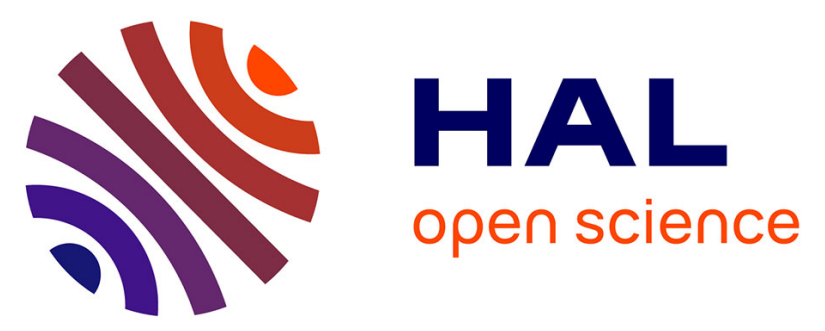

\title{
Cationic-Surfactant-Coated Mica Surfaces below the Critical Micellar Concentration: 1. Patchy Structures As Revealed by Peak Force Tapping AFM Mode Patrick Kékicheff, Christophe Contal
}

\section{To cite this version:}

Patrick Kékicheff, Christophe Contal. Cationic-Surfactant-Coated Mica Surfaces below the Critical Micellar Concentration: 1. Patchy Structures As Revealed by Peak Force Tapping AFM Mode. Langmuir, 2019, 35 (8), pp.3087-3107. 10.1021/acs.langmuir.8b03781 . hal-02404906

\author{
HAL Id: hal-02404906 \\ https://hal.science/hal-02404906
}

Submitted on 11 Oct 2021

HAL is a multi-disciplinary open access archive for the deposit and dissemination of scientific research documents, whether they are published or not. The documents may come from teaching and research institutions in France or abroad, or from public or private research centers.
L'archive ouverte pluridisciplinaire HAL, est destinée au dépôt et à la diffusion de documents scientifiques de niveau recherche, publiés ou non, émanant des établissements d'enseignement et de recherche français ou étrangers, des laboratoires publics ou privés. 
Cationic surfactant-coated mica surfaces below the critical micellar concentration:

Patchy structures as revealed by Peak Force Tapping AFM mode

\author{
Patrick Kékicheff* and Christophe Contal \\ Université de Strasbourg, C.N.R.S. Institut Charles Sadron \\ 23 rue du Loess, 67034 Strasbourg Cedex 2, France
}

*Corresponding author: patrick.kekicheff@ics-cnrs.unistra.fr

\author{
Manuscript submitted to Langmuir
}

\begin{abstract}
The morphology and structure of the self-assembled surfactant aggregates at the solid-liquid interface remain controversial. For the well studied system of cationic cetyltrimethylammonium bromide $\left(\mathrm{C}_{16} \mathrm{TAB}\right)$ adsorbed onto the opposite negatively charged atomically smooth mica surface, a variety of surface aggregates have been previously reported: AFM imaging pointing to cylinders and surface micelles as opposed to mono/bilayered-like structures revealed by neutron and X-ray reflectometry, NMR, spectroscopic techniques, and numerical simulations. In order to reconcile with the latter results, we revisit the morphometry of the $\mathrm{C}_{16} \mathrm{TAB}$-coated mica surfaces using the recent Peak Force Tapping (PFT-AFM) mode that allows fragile structures to be imaged with the lowest possible applied force. The evolution of the structural organization at the mica-water interface is investigated above the Krafft boundary over a wide concentration range (from 1/1000 cmc to $2 \mathrm{cmc}$ ) after long-equilibration times to insure thermodynamic equilibrium. A complex but fairly complete picture has emerged: At very low concentrations the $\mathrm{C}_{16} \mathrm{TAB}$ surfactants adsorb as isolated molecules before forming small clusters. Above 1/140 cmc, monolayeredlike stripes are formed. As the concentration is increased, a connected network of these patches covers progressively the mica substrate. Above $1 / 80 \mathrm{cmc}$, bilayered-like patches build on top of the underlying monolayer and ultimately a complete bilayer (at about half the $\mathrm{cmc}$ ) covers the entire mica substrate. Thanks to the less invasive PFT-AFM imaging mode, our observations agree not only with the theoretical predictions and numerical simulations, but also reconcile, at last, the direct observations by means of the AFM imaging technique with the results obtained with other techniques.
\end{abstract}

Keywords: surfactant adsorption; Peak Force Tapping AFM; mono/bilayered-like structures; surfactant patches; Cetyltrimethylammonium bromide; mica substrate 


\section{Introduction}

Understanding the characteristics of adsorbed amphiphilic molecular structures at the solid-aqueous interface and their properties is important to many chemical and industrial applications and processes, ranging from detergency, dispersants or stabilization of suspensions, ${ }^{1}$ paint and coating technology, surface conditioning (hair care formulations, sunscreens, make-up products, fabrics, dyeing, etc.), ${ }^{2}$ filtration, ${ }^{3}$ nanoparticle synthesis ${ }^{4,5}$ and nanoparticle decoration for imparting specific functions (catalysts, biomarkers, therapeutics) ${ }^{6}$ remediation of contaminated soils, ${ }^{7,8}$ lubrication, ${ }^{9}$ ore flotation, ${ }^{10}$ to enhanced oil recovery ${ }^{11}$ (for which surfactant adsorption is detrimental to surfactant-enhanced oil recovery) and prevention of activity loss of protein-based pharmaceuticals. ${ }^{12}$ The way the surfactant molecules interact with the substrate whether due to electrostatic attraction, hydrogen or covalent bonding, or hydrophobic forces, will determine the structure and morphology of the adsorbed film, and thereby how the surface properties of the substrate are modified when it gets coated. At a hydrophobic surface, the adsorption is in the form of a monolayer and is similar to that observed at the air-solution interface. ${ }^{13}$ In contrast, at a hydrophilic surface surfactant adsorption is highly cooperative and is in the form of surface aggregates. ${ }^{1}$ As an alternative to monolayer and bilayer formation, has been reported the formation of various surface aggregates. These include hemimicelles (composed of associated monomers adsorbed to the substrate with the headgroups next to the surface and the hydrocarbon chains in contact with the aqueous phase) ${ }^{14}$ and admicelles (surface aggregates composed of surfactant molecules oriented with headgroups facing both toward the substrate and into the solution; the alkyl tails form a hydrophobic core between the heads). ${ }^{15-17}$ For ionic surfactants adsorbed onto oppositely charged substrates, the cooperative nature of the adsorption is reinforced by electrostatic attraction. Model hydrophilic surfaces, such as amorphous silica, crystalline silicon or quartz, or mica, have been studied.

Despite progress in the understanding of surfactant adsorption to a charged interface, many issues are still unresolved. ${ }^{18,19}$ The exact mechanism of surfactant adsorption remains difficult to quantify due to an inability to reveal the precise structure of the incomplete film. The question regarding the shape, the structure, and the organization of surfactant aggregates adsorbed onto a substrate has been a thorny one.

Molecular simulations have shown promising outcomes but also some limitations. ${ }^{20}$ The latter are due to time and length scales accessible much smaller than those encountered in experimental observations. For instance, the simulation times conducted at atomistic resolution are at most on the order of hundreds of nanoseconds, thereby much shorter than the typical times required for surfactants to exchange between surface and bulk aggregates, which are on the order of microseconds. Monte Carlo and coarse-grained simulations allow savings in computational time by reducing either possible configurations or the number of details that are explicitly considered. These models have thus allowed general guidelines to be set, such as guiding principles regarding the physical behavior of self-associating systems, but are in general not able to capture the details of molecular behaviors. However, limitations are inherent in models based at the expense of atomic-level accuracy, as those are unable to describe the solvent structure appropriately and often deal with time scales some orders of magnitude smaller than the hours or even longer times that are required to reach proper equilibration as observed experimentally.

On the experimental side several techniques have been used to infer the morphology, the structure and the organization of the adsorbed surfactants on a substrate (see the recents reviews ${ }^{18,19,21,22}$ ). The wealth of experimental data reported for decades has been extremely beneficial for allowing the researchers to possibly identify the molecular phenomena responsible for the variety of observations between different surfactants, substrates, and 
solution conditions, but also for allowing the limitations between different techniques to be better interrogated as some discrepancies between observations have not been solved yet. ${ }^{19}$ Atomic force microscopy (AFM) has thus emerged as one of the most suitable techniques for directly imaging adsorbed surfactant structures on a substrate. Most of the meaningful AFM data have been obtained above the critical micellar concentration $(\mathrm{cmc})$, as it is easier to image aggregates when a repulsive force is provided by the surfactant layer having headgroups facing solution. ${ }^{21}$ Conversely, in the low concentration range much below the cmc, imaging small adsorbed aggregates and determining their lateral organization on the substrate are rendered more difficult for at least three reasons: loose adsorbed molecules and isolated small aggregates may be displaced or even swept away upon scanning, deformation of fragile objects may occur upon imaging, and the finite size of the AFM tip (on the order of $10 \mathrm{~nm})$ tends to overestimate the lateral dimensions of the imaged objects. ${ }^{23}$ As a consequence whereas most AFM studies have depicted the situation at full surface coverage, structural information on the adsorbed layer at lower surface coverage is limited: AFM does not give resolved images at low surface densities and only a limited number of AFM investigations have been reported in the regime much below the cmc. Other techniques such as neutron and X-ray reflectometry have also been largely used, but again rare are the reports investigating substrates in contact with surfactant solutions at low bulk concentrations. Indeed, contrary to a high resolution in the direction perpendicular to the surface allowing the overall thickness of the adsorbed layer to be inferred, reflectometry techniques provide limited in-plane resolution. Thus the analysis of the measured reflectivity profiles may become model-dependent when one attempts to take into account any structural heterogeneity along the adsorbed film resulting from the lateral organization of the adsorbed aggregates. Therefore, the conclusions are not unequivocal, although it should be noted that recent improvements in radiation sources plus the combination of X-ray and neutron scattering experiments are allowing a greater differentiation between structural models.

Probably one of the most studied systems so far has been the hydrophilic muscovite mica substrate, negatively charged in aqueous solutions, ${ }^{24-26}$ onto which the opposite charged cationic quaternary alkyl ammonium bromide surfactants $\mathrm{C}_{n} \mathrm{TAB}(n=8-20)$ adsorb from bulk solutions. Indeed, due to its perfect (001) cleavage allowing easily achievable creation of large macroscopic areas of smooth surface on atomic scale, mica is an ideal substrate ${ }^{27}$ for AFM, ${ }^{28}$ Surface Force Apparatus (SFA), ${ }^{29}$ and electron microscopy ${ }^{30}$ observations. Among the $\mathrm{C}_{n} \mathrm{TAB}$ family, the cetyltrimethylammonium bromide $\left(\mathrm{C}_{16} \mathrm{TAB}\right)$ adsorption on mica has been characterized by many experimental techniques, including AFM, ${ }^{31-40} \mathrm{SFA},{ }^{41-51}$ neutron ${ }^{52}$ and X-ray reflectivity, ${ }^{53,54}$ X-ray photoelectron spectroscopy (XPS), ${ }^{55}$ and simulation studies. ${ }^{56}$ All these studies closely agree in their results and conclusions in the regime above the cmc $\left(\sim 0.9 \mathrm{mM}\right.$ at $\left.25{ }^{\circ} \mathrm{C}{ }^{57,58}\right)$ once the thermodynamic equilibrium is attained: the muscovite mica surface is entirely covered by a $\mathrm{C}_{16} \mathrm{TAB}$ bilayer whose thickness (around 3.2 $\mathrm{nm}$ ) indicates that the surfactant molecules are either interdigitated or tilted (since the thickness of a bilayer including the inner and outer headgroups of two fully extended $\mathrm{C}_{16} \mathrm{TAB}$ molecules would be about $4.7 \mathrm{~nm}^{54}$ ).

However, in the regime below the cmc, structural information on the adsorbed layer much has appeared to be limited and confused: the literature provides a variety of contradictory observations. ${ }^{19}$ Thus, AFM images were interpreted as adsorbed spherical admicelles, hemi-cylindrical structures, fully or defective cylindrical aggregates with possibly flattened bottom and with lengths from short rods to long ones, meandering stripes, etc., for $\mathrm{C}_{n}$ TABs below the cmc adsorbed on different substrates such as mica, ${ }^{31,32,36,37,40,59-63}$ glass or silica, ${ }^{64}$ and graphite. ${ }^{65}$ Conversely, other techniques have shown that the adsorption occurs in the form of patchy bilayered-like aggregates, differing from the morphology by AFM studies. Thus, by neutron reflectometry the structures of $\mathrm{C}_{16} \mathrm{TAB}$ at flat solid/liquid interfaces, such as 
silica, ${ }^{66-69}$ quartz $^{70}$ and silicon ${ }^{67}$ were investigated. For instance, the surface coverage of adsorbed $\mathrm{C}_{16} \mathrm{TAB}$ layers on silica was found to increase from $35 \%$ at $1 / 3 \mathrm{cmc}$ to $80 \%$ at $2 / 3$ cmc; importantly, the thickness of the surfactant layer remains essentially constant in the range of 2.8-3.4 nm down to concentrations as low as $1 / 100 \mathrm{cmc},{ }^{67,70}$ a value that appears larger than a fully extended $\mathrm{C}_{16} \mathrm{TAB}$ molecule but significantly less than twice that length, and hence consistent with either a defective bilayer or flattened micelles on silica ${ }^{66}$ Recent Xray reflectometry measurements at the highly charged mica-water interface supplement these observations in the surfactant concentration range below the cmc $(>1 / 10 \mathrm{cmc}){ }^{54} \mathrm{In}$ agreement with direct force measurements both on mica ${ }^{45,51,71}$ and on glass or silica, ${ }^{72-74}$ all the reflectometry studies support the idea that $\mathrm{C}_{16} \mathrm{TAB}$ adsorption occurs via the formation of mono/bi-layered-like fragments, and the surface is not fully covered when the substrate is immersed in surfactant solutions well below the cmc. Interestingly, the same conclusions about solids covered heterogeneously by surfactant patches are obtained not only on small platelets, but also on curved surfaces, either colloidal particles, or of the inverse geometry, such as pores in solid materials, using different techniques ranging from small angle scattering ${ }^{75,76}$ and X-ray diffraction, ${ }^{77}$ spectroscopic techniques (infrared, ${ }^{78-82}$ fluorescence quenching, ${ }^{83}$ Raman, ${ }^{82,84,85}$ sum frequency generation (SFG) spectroscopy ${ }^{86}$ ) to NMR techniques (see ref. 87-89 and references therein). Thus, the structural information gained from the dynamic NMR leads to the same structural picture as obtained from reflectometry techniques: there is formation of anisotropic surface aggregates whose shape is disc-shaped micelles or bilayer-like patches. ${ }^{87,90,91}$ For these high-charge-density surfaces such as mica and silica, calorimetric studies are also consistent with the interpretation of small discrete aggregates at the lowest adsorption densities and bilayer formation in the later stage of adsorption. ${ }^{92,93}$ Remarkably, the surface aggregate shape does not change significantly as a function of surface coverage: only the number of defects in the distorted and disrupted bilayered-like film increases as the surface coverage gets low and conversely a complete bilayer is found at the adsorption plateau. Theoretical modeling ${ }^{94}$ and numerical simulation studies $^{56,95,96}$ are in agreement with the conclusions drawn by all the preceding quoted techniques (neutron and X-ray reflectometry, SFA, IR, Raman, SFG, NMR, calorimetry), and hence in contrast to the organization of surfactant aggregates proposed in the AFM literature, shown as never energetically favored. Rather, common to each surface structure is monolayered-like aggregates oriented with surfactant head groups in contact with the hydrophilic surface. On top of these local monolayers additional surfactants with opposite molecular orientation are observed, and hemispheres, hemicylinders or another monolayer, that is patchy bilayered-like aggregates (admicelles) may form. These aggregates are the result of local 2D condensations on the homogeneous or heterogeneous surface characterized by a surface topography intermediate between "patchwise" and "random". The areas covered by the surfactant film lead to ultimate heterogeneous surfaces that are partially correlated.

In summary, the morphology and structure of the self-assembled surfactant aggregates at solid/liquid interfaces has so far remained controversial, ${ }^{19}$ and thereby the adsorption mechanism is yet to be fully understood, despite extensive related studies. In general, NMR, SFA and comprehensive reflectometry results suggest patches of local monolayers or bilayered-like structures whilst in contrast AFM studies interpret the images with a variety of surface morphologies. It has been noted that the discrepancies cannot be explained by different time frames between reflectometry and AFM experiments. ${ }^{54}$ Rather it has been suggested that the surface aggregates observed with AFM might be transient and induced by both the applied load of the AFM tip on the fragile surfactant structure adsorbed on the substrate, in addition to the interactions between the AFM tip and the surface layer. ${ }^{54}$ Clearly more AFM experiments are called for in order to reconcile with the conclusions inferred by 
the other techniques and the numerical simulations: this is the goal of our present investigation.

So far, to analyze surfactant films adsorbed at the solid-liquid interface the most often applied direct imaging techniques have been the contact atomic force microscopy mode for long, and then the amplitude modulation atomic force microscopy (AM-AFM, also known as tapping mode or intermittent contact mode atomic force microscopy $\left.{ }^{23}\right)$. Because the tip's interaction with the sample surface is relatively gentle, this latter mode has been rapidly preferred to the former one, as it was recognized that damage of fragile samples is more likely to occur when operating the contact mode under large loading forces. While the presence and adsorbed surfactant aggregates / areas can be unequivocally detected, it remains unclear how much the a priori invasive nature of AFM affects the apparent shapes and dimensions of the observed objects. Indeed, AM-AFM suffers from the drawback that it is difficult to control the normal force acting on the sample surface as the technique detects changes in the amplitude of the resonance frequency oscillation of a cantilever. ${ }^{23}$ In addition the pronounced lateral forces involved in the scanning by the tip action are prone to the displacement ${ }^{97}$ and eventually removal of materials weakly adsorbed on the substrate. They even may induce morphometry transformations of the surface aggregates as discussed by Briscoe et al. ${ }^{19,54}$ This was also noted in works dealing with the imaging of other fragile objects (such as nanobubbles): the force exerted by the tip alters the apparent dimensions to such an extent that it cannot be neglected. ${ }^{98}$ This means that there is a high likelihood of perturbing any soft sample, particularly highly deformable surface aggregates during AM-AFM measurements. ${ }^{37}$ The true shapes and sizes in the absence of an applied force could be obtained by extrapolating the AFM height data to conditions where the oscillations of the cantilever would have zero amplitude, but such conditions are generally physically impossible to obtain during an AM-AFM scanning.

However and fortunately, recent advances in AFM technology circumvent these limitations and enable researchers to record quantitative images of fragile objects with an improved resolution and a better reproduction of the sample profile. Thus recently, a novel AFM imaging mode called peak force tapping (PFT-AFM) has been applied to many systems with success for imaging delicate objects such as biological entities, ${ }^{99-101}$ nanobubbles, ${ }^{102-104}$ polymer films and brushes, ${ }^{105}$ but also for measuring mechanical properties at the nanoscale using its derivative technique, referred to as peak force quantitative nanomechanics (PFQNM). ${ }^{106}$ In PFT-AFM the probe and sample are intermittently brought into contact for a short period, substantially eliminating lateral and shear forces during sample motion in the $(x, y)$ plane. Typically the tip is oscillated in the vertical direction at a frequency of at least one order smaller than the tip resonance to avoid resonant effects. The $z$-piezo is driven with a sinusoidal rather than a triangular waveform in conventional force-distance curves. This oscillating system minimizes parasitic coupling so that a far more sensitive force control than AM-AFM can be accomplished (at least 3 orders magnitude). This allows direct force control of damaging lateral forces, which is very useful for the structural imaging of soft samples. Unlike in AM-AFM, in which the feedback loop keeps the cantilever vibration amplitude constant, PFT-AFM controls the maximum force applied by the tip. The synchronized peak force control eliminates the complications due to cantilever dynamics, as well as the complications induced by cantilever resonance and its multiple harmonics modes encountered in AM-AFM. Furthermore, because the PFT-AFM mode uses the interaction force directly as the feedback control parameter, constant drift of the cantilever deflection due to thermal or other system factors that previously made accurate force control impossible even in contact mode, is now rendered negligible. In PFT-AFM, the system re-establishes the non-interacting baseline by moving the probe far from the sample in each interaction period. This process allows accurate determination of the interaction force every time the probe interacts with the 
sample. An added benefit of PFT-AFM mode is that each cycle of the vertical movement produces a force curve, or multiple force curves at each pixel, allowing simultaneous acquisition and mapping of height and mechanical property data. Therefore topographic, adhesion, and mechanical maps are generated to a good resolution (up to 4096 pixels x 4096 pixels) with imaging speed higher than in the conventional tapping mode. As its name indicates, the peak force tapping mode generates and analyzes each and every individual force curve, then measures and controls the AFM based on the corresponding peak interaction forces during each instance of the tip tapping on the sample.

Taking advantage of the PFT-AFM mode that makes it possible to better control the interactions between the AFM tip and the substrate, we have investigated the film morphology for the cationic $\mathrm{C}_{16} \mathrm{TAB}$ surfactant adsorbed on the oppositely charged mica surface. In this first contribution of a series of articles on this system, we report how the structural patterns evolve over a large concentration range spanning from concentrations as low as $1 / 1000 \mathrm{cmc}$ up to $2 \mathrm{cmc}$. The mica substrates, bathed at $25^{\circ} \mathrm{C}$ above the $\mathrm{Krafft}$ boundary, were left equilibrated for a long time ( $24 \mathrm{~h}$ or longer) to insure the thermodynamic equilibrium. Through the study of the effects of surfactant concentration on the adsorption a complex but fairly complete picture emerged for the film structure and the organization of the surface aggregates.

\section{Materials and methods}

\section{Materials}

Prior to use, all glassware was submitted to a thorough cleaning procedure, through the use of a solution of ammonium persulfate in pure sulfuric acid, a copious rinse with ultrapure water, and the drying with nitrogen. Ultra pure water was obtained from a commercial Millipore purification system (MilliQ Gradient system). The end product had a resistivity of $18.2 \mathrm{M} \Omega . \mathrm{cm}^{-1}$, a surface tension $72.5 \mathrm{mN} / \mathrm{m}$ at $22.0^{\circ} \mathrm{C}$, a pH about 5.7 (due to equilibration with dissolved atmospheric $\mathrm{CO}_{2}$ ), and showed no bubble persistence.

All surfactant solutions were freshly prepared prior to each series of experiments. The hexadecyltrimethylammonium bromide $\left(\mathrm{CH}_{3}\left(\mathrm{CH}_{2}\right)_{15} \mathrm{~N}^{+}\left(\mathrm{CH}_{3}\right)_{3} \mathrm{Br}-\right.$, noted hereafter $\left.\mathrm{C}_{16} \mathrm{TAB}\right)$ was obtained from B.D.H. (> 98\% assay) and used after double recrystallization from a 70/30 acetone/ether mixture. Atomic and ion analysis confirmed that the samples had the correct composition to $1 \%$ or better. No chloride was detected in the batch $(<\mathrm{ppm})$. Several series of $\mathrm{C}_{16} \mathrm{TAB}$ solutions were carefully prepared in a clean room (class 10000) to explore a large concentration range below the $\mathrm{cmc}$ (from $2 \times 10^{-3} M$ to $10^{-6} M$, i.e. from about $2 \mathrm{cmc}$ down to $1 / 1000 \mathrm{cmc}$ ). The most concentrated samples were prepared simply by weighing each constituent $\left(\mathrm{C}_{16} \mathrm{TAB}\right.$ and water) into a glass-container which was then hermetically sealed and allowed to equilibrate above the Krafft boundary (defined as the crystal solubility boundary of surfactant ${ }^{107}$ ) at $25^{\circ} \mathrm{C}{ }^{58,108,109}$ for $48 \mathrm{~h}$ with frequent shaking and several ultrasonications. The lowest concentrated surfactant solutions were obtained by water dilution of the most concentrated ones. As a precaution against dissolved silicate and other contamination, water drawn through the MilliQ unit was stored in a laminar flow cabinet in a stopped flask for no longer than 24 hours. Note also that the water which is saturated with nitrogen after removal from the MilliQ unit was degased for an hour under vacuum beforehand and sample preparation. This caution also reduces the possibility of small bubbles being introduced into the AFM chamber. Prior to injection into the AFM liquid chamber the surfactant solutions were sonicated once more to prevent the presence of any aggregate in the bulk. Adjustment of the $\mathrm{pH}$ to a constant value was not sought. This minimizes the number of chemicals in the unbuffered ultrahigh purity water and the surfactant solutions at ambient $\mathrm{pH}(\sim 5.7)$ can be 
considered pure.

Large sheets of brown muscovite mica (chemical formula: $\left.\mathrm{KAl}_{2}\left(\mathrm{Si}_{3} \mathrm{Al}\right) \mathrm{O}_{10}(\mathrm{OH})_{2}\right)$ of about $0.3 \mathrm{~mm}$ in thickness from Bahir, India, were obtained from JBG-Metafix, France. The high-quality grade ASTM-V-1 was chosen: the sheets are clear, hard, of uniform color, nearly flat, free of all stains, foreign inclusions, cracks, and other, similar defects.

\section{AFM experiment}

\section{Procedure}

The AFM liquid cell and all other hardware, tubing, fittings that came in contact with surfactant solutions were cleaned before each experiment with absolute ethanol, and rinsed copiously with Milli-Q water, and then blown dry with nitrogen. Substrates were freshly prepared immediately before injection of the $\mathrm{C}_{16} \mathrm{TAB}$ solution into the AFM liquid cell. Thin sheets of muscovite mica were attached onto stainless disks (13 mm diameter) using Loctite $\mathbb{R}$ cyanoacrylate adhesive (Henkel AG, Pratteln, Switzerland). Mica was stripped with adhesive tape along the basal plane in order to obtain atomically smooth, freshly cleaned (top) surfaces. Calibration in air of sensitivity and spring constant was then performed as follows: first, the tip is engaged on the mica, a representative force curve is captured, and the deflection sensitivity calculated. Second, the tip is withdrawn and the spring constant is calculated by using a thermal tune (a sweep in frequency allows finding the resonance peak). $\mathrm{C}_{16} \mathrm{TAB}$ solutions of known molarity were then injected into the AFM fluid cell. It was also checked after every assembly of the AFM liquid cell with its O-ring that adequate positioning was achieved to prevent any possible drift upon scanning. The solutions were left to equilibrate at the temperature of the experiment $\left(25^{\circ} \mathrm{C}\right.$; above the Krafft boundary). Equilibration for up to $24 \mathrm{~h}$ was necessary to obtain reproducible results in the low concentration regime $\left(<10^{-4} M\right)$. For higher concentrations, several hours of equilibration $(<12 \mathrm{~h})$ were sufficient. The $\mathrm{C}_{16} \mathrm{TAB}$ concentration range was explored by carrying out at least two independent experiments for each concentration allowing the reliability of the results to be increased.

Prior to the series of experiments, the AFM nanopositioner of the Multimode 8 AFM instrument equipped with a NanoScope V controller 64 bits (Veeco Metrology, Santa Barbara, CA) was calibrated. Indeed, independently of the fact that the apparatus is currently placed on a damping system to minimize perturbations arising from mechanical vibration, piezoelectric nanopositioners exhibit positioning error measurements due to environmental effects and aging. Lateral and height scale calibration were performed to minimize uncertainty due to different kinds of lateral and height errors. ${ }^{110}$ To calibrate the scanner, several repeatability tests were performed against a reference grid with a $1 \mu \mathrm{m}$ pitch grating. The calibration of the scanner was said to be valid when the repeatability test results were in agreement with the nominal values of the reference materials within the specified uncertainties $(<5 \%)$.

\section{AFM imaging}

PeakForce tapping mode AFM images were collected using V-shaped nitride coated silicon tip on a nitride cantilever (Bruker ScanAnyst-Fluid, nominal spring constant $k=0.7$ $\mathrm{N} / \mathrm{m}$, resonance frequency between 130 and $150 \mathrm{kHz}$, sensitivity about $0.2 \mathrm{~nm} / \mathrm{V}$ ). In the PFT-AFM mode the sample was oscillating at $2 \mathrm{kHz}$ (off resonance), the PeakForce tapping amplitude was set to $100-150 \mathrm{~nm}$ and the peak force threshold was optimized and kept constant for each image. The applied force was kept at the lowest possible value (typically, in the range between 0.2 and $0.8 \mathrm{nN}$ depending on the area size scanned) to minimize the interactions between the tip and the surface, to avoid damaging the sample or wearing the tip. Scanning speed and feedback gains were carefully adjusted in order to limit errors from response time limitations and until trace and retrace line were superimposed. The images were 
captured in the trace direction with a linear scanning rate of $1 \mathrm{~Hz}$ with scan resolution of minimum 512 samples per line. Typically a $1 \mu \mathrm{m}$ x $1 \mu \mathrm{m}$ image $(512$ pixel x 512 pixel $)$ recorded at a scan rate of $1 \mathrm{~Hz}$ took $8 \mathrm{~min}$.

For comparison, a few images were also acquired by means of the conventional tapping AFM mode (amplitude modulation atomic force microscopy, AM-AFM) in liquid. These investigations used standard microfabricated silicon cantilevers (cantilevers with spring constant of $5 \mathrm{~N} / \mathrm{m}$, resonance frequency of about $75 \mathrm{kHz}$ in air, nominal radius of curvature of the tip, 5-10 nm), although a few AM-AFM experiments were also performed with the same tip used in the PFT-AFM mode. Taking advantage of a sequential use of the two modes, such investigations allowed direct comparison to be established for the same solution and adsorption conditions strictly performed during the same experiment.

\section{AFM image analysis}

Because the mica substrate is glued to the sample mount, a small amount of tilting of the surface is inevitable. This setup error represents only kinematic movement that is irrelevant to surface inherent topography, and was removed in image preprocessing using the NanoScope ${ }^{\mathrm{TM}}$ Analysis software (Bruker). Thus all topographic AFM images were flattened by line-by-line subtraction of $0^{\text {th }}$ to $2^{\text {nd }}$ order polynomials evaluated on flat substrate regions. Once these original and global AFM images were processed, the useful images $(512 \times 512$ pixel size) of the structure were extracted and converted into black and white images. The digitized images were analyzed with imageJ analysis software following an edge detection procedure to determine the surface coverage. Several techniques were combined, such as filtering (background correction and Gaussian smoothing for removing detail and noise), edge detection methods (Laplacian of Gaussian and zero-crossing operators), and adaptative threshold to locate the boundary (or edge) of each domain considered as composing the adsorbed surfactant film.

\section{Results}

The aims of this work were to characterize the $\mathrm{C}_{16} \mathrm{TAB}$ films adsorbed on hydrophilic mica surfaces by exploring the differences in structure and organization, surface coverage, etc., along the entire adsorption isotherm at $25{ }^{\circ} \mathrm{C}$ above the Krafft boundary. With its direct imaging character, AFM allows the solid-liquid interface in contact with the aqueous surfactant solutions to be visualized. Nevertheless, since AFM is a priori an invasive technique and is prone to provide apparently quantitative data even if the measurement conditions preclude this (especially in the case of soft structures), great care was taken to perform the measurements with an adequate protocol. In particular, all the results presented in this first contribution were obtained after long equilibration times to overcome any variation due to kinetics effects. This strict protocol removes any uncertainty as to the record of microstructural morphometry at the thermodynamic equilibrium. In the present work we do not report any observation during the film build-up (otherwise explicitly mentioned). As a result the shapes and sizes of the surface aggregates, the rearrangements and changes in the surface features and in the film organization, the topological transformations, are all those induced by the effect of varying the bulk surfactant concentration of the aqueous solution in contact with the mica surface. Thanks to data consistency in the manner to collect them the comparison becomes facilitated. As a result one can monitor the evolution of the different film morphologies formed on mica against the aqueous solutions of a broad $\mathrm{C}_{16} \mathrm{TAB}$ concentration range $(1 / 1000 \mathrm{cmc}$ to $2 \mathrm{cmc})$. 
As it was also deemed worthwhile to compare the images obtained with the recent, less invasive, PFT-AFM mode to that measured in the previous modes (such as the AMAFM), we first present our results for a sample chosen in the middle range of the spanned concentration. Then we will describe the evolution of both the structure and the morphology of the adsorbed films as a function of the $\mathrm{C}_{16} \mathrm{TAB}$ concentration. The choice of this presentation order allows us to establish first which reliable information can be inferred from the images. In doing so, we set the framework for the rest of the investigation and underline which relevant structural data can be measured.

\section{Organization of the adsorbed film at 1/100 $\mathrm{cmc}$}

As stated in the Introduction, conventional tapping AFM mode (AM-AFM) has so far been the most common AFM mode to image surface structures of adsorbed surfactants on substrates. Therefore, in this section, we briefly compare our results obtained on $\mathrm{C}_{16} \mathrm{TAB}$ adsorption on mica in PFT-AFM mode with data acquired using AM-AFM. As an illustration for these two modes we present the images of a $\mathrm{C}_{16} \mathrm{TAB}$-coated mica surface immersed in an aqueous $\mathrm{C}_{16} \mathrm{TAB}$ solution at $\approx 1 / 100 \mathrm{cmc}$ after $24 \mathrm{~h}$ equilibration (Fig.1). This concentration lies in the middle of the investigated surfactant range below the cmc. For this peculiar experiment, the same AFM tip was used in both modes: the PFT-AFM mode was launched before the AM-AFM mode was performed. Figure 1 shows the comparison of $1 \mu \mathrm{m} \times 1 \mu \mathrm{m}$ representative AFM topographies of the $\mathrm{C}_{16} \mathrm{TAB}$-coated mica film as seen with these two modes. These areas are not necessary the same ones that were scanned later with AM-AFM, but their sizes and morphologies were of the same range. The exemplary images scanned in both modes reveal that the thin $\mathrm{C}_{16} \mathrm{TAB}$ coating on mica is composed of bare regions of the substrate coexisting with surfactant layered structures.

The AM-AFM image presented as height data (Fig. 1a) is in good agreement with the images that were reported for $\mathrm{C}_{16} \mathrm{TAB}$ adsorbed on mica ${ }^{32}$ and on silica ${ }^{64}$ below the cmc. In these works a "soft contact mode" was used (in which surfactant adsorption on both the tip and the substrate allows imaging using electrical double layer repulsion without the tip physically contacting the sample $\left.{ }^{65,111}\right)$. Because the reported images in those works are presented as deflection images (showing the error in the feedback signal) no height profile can be inferred. Conversely our images using the conventional tapping mode suggest an adsorbed layer height of about 1.5-2.0 nm (Fig.1a). The adsorbed $\mathrm{C}_{16} \mathrm{TAB}$ layer has a patchy appearance composed of quasi-discrete structures distributed uniformly across the surface. Unfortunately the poor contrast quality of the images collected with this mode prevents a more precise analysis of the structural features. This limitation was already pointed out by the authors of the pioneering works: "the image contrast is not as great as typically observed for surface aggregates above the cmc. This is most likely due to the lower density adsorbed layer, which is more easily displaced with the imaging tip". ${ }^{64}$ Furthermore, some image distortions may occur due to the non-spherical probe tip shape and the poor contact with the sample during scanning with very high setpoint ratios, resulting in poor tracking of the surface contour once the tip has traversed the fragile object. ${ }^{12}$

Although it is clear that the conventional taping mode affects the topographical features, some additional information about the physico-chemical properties of the thin $\mathrm{C}_{16} \mathrm{TAB}$ coating on mica can nevertheless be inferred through phase imaging. The latter is routinely performed simultaneously to intermittent-contact mode (tapping-mode), as it consists in recording the phase lag between the sinusoidal driving force and the oscillation of the AFM tip. ${ }^{23}$ Phase images reflect differences in energy dissipation and are thought to probe variations in adhesive and mechanical properties over heterogeneous surfaces, depending on 
the tapping strength. A possible viscoelastic contribution can also be considered. Thus tapping mode with phase imaging has been used as a tool for compositional mapping. ${ }^{23}$ Figure $1 \mathrm{~b}$ shows the phase map of the region recorded simultaneously with the topographic image (Fig.1a) in the AM-AFM mode. A moderate variation of the phase shift $\left(\approx 10^{\circ}\right)$ is observed over the domains during phase imaging. Because the phase contrast can be associated to different visco-elastic properties of the sample surface, and more generally to different tip-surface interactions, this phase shift indicates that the domains are soft and different in nature from the bare mica solid surface. A typical view of the phase shift profile across the scanned area is shown in Fig.1b. It can be noticed a weak contrast between different $\mathrm{C}_{16} \mathrm{TAB}$ layers (the phase value is almost constant around a few degrees across virtually the whole distance of the layered domains).
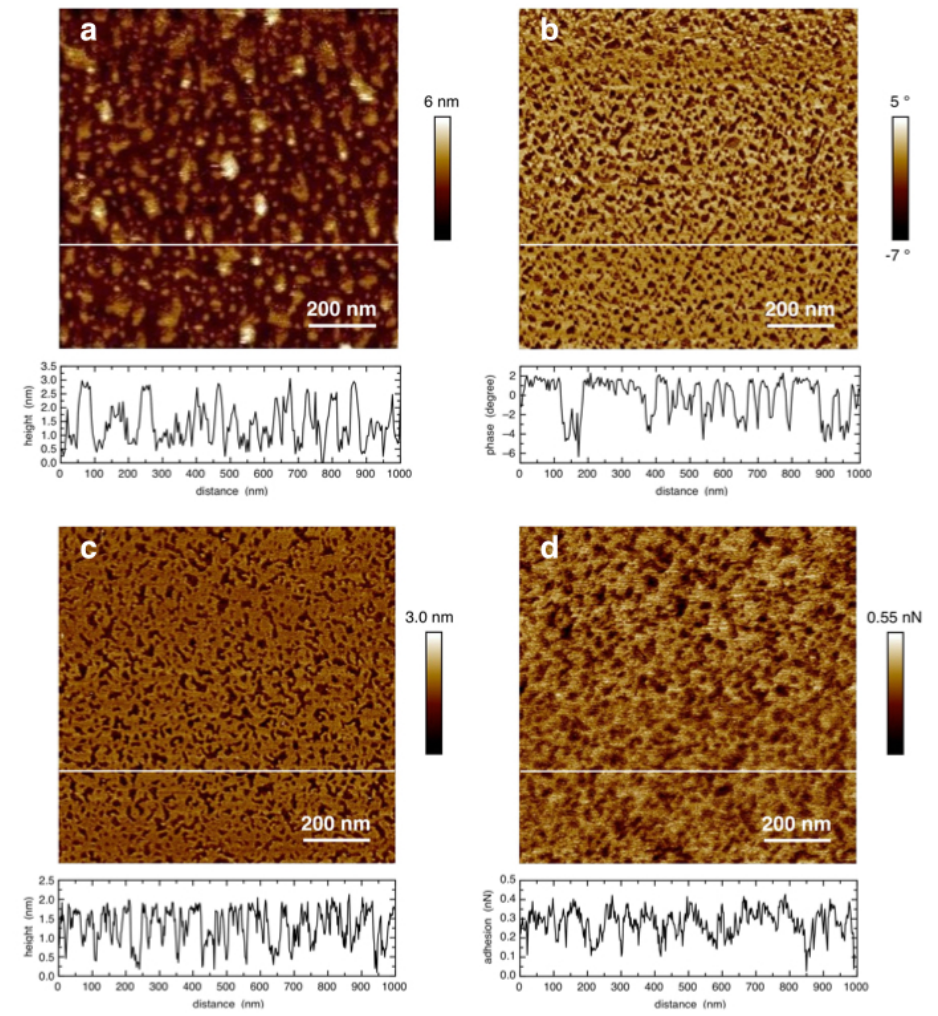

Figure 1: Comparison of the AFM images obtained by means of the conventional tapping mode and of the recent PeakForce mode scanning a $C_{16} T A B$-coated mica surface immersed in a $C_{16} T A B$ solution at $\approx 1 / 100 \mathrm{cmc}$ and equilibrated for $24 \mathrm{~h}$ at $25{ }^{\circ} \mathrm{C}$. Simultaneously recorded topographic (height; a) and phase maps (b) in conventional Tapping mode (AM-AFM) with height and phase profiles along the horizontal white lines. The difference in the phase value is interpreted as the presence of hydrophobized domains surrounded by bare mica regions. Simultaneously recorded topographic (height; c) and adhesion maps (d) in PeakForce Tapping mode (PFT-AFM) with height and adhesion profiles along the horizontal white lines, revealing bare mica areas surrounded by adsorbed $C_{16} T A B$ regions regular in thickness $(1.7-1.9 \mathrm{~nm})$.

This suggests that the surfactant domains behave similarly despite their (small) variations in thicknesses (as observed in the topographic image): the $\mathrm{C}_{16} \mathrm{TAB}$ molecules may be in different phases or conformations (tilted, upright) but compose the different layered domains with a balance between hydrophobicity and hydrophilicity that remains almost the same over the thin $\mathrm{C}_{16} \mathrm{TAB}$-coated mica film. While it is straightforward to compare the phase lags of different forms of materials (the bare substrate mica and the adsorbed film), the comparison of phase signal of the same materials (the $\mathrm{C}_{16} \mathrm{TAB}$ molecules present in different 
conformations or adsorbed phases), in particular the interpretation of weak phase contrast is not obvious, and would require less qualitative application of phase imaging technique and models. This shortcoming together with the fact that the AM-AFM mode does not allow direct control of the force exerted have lead us to employ the novel and recent imaging approach, less invasive, namely the PFT-AFM mode, in order to investigate the microstructuration of the fragile adsorbed surfactant layer with the lowest possible applied force.

In the PFT-AFM mode it is the force exerted on the sample rather than the amplitude of the resonating cantilever that is used as the AFM feedback parameter during imaging. The so-called adhesion, dissipation and deformation images were acquired simultaneously with the height images. Exemplary images for the height and adhesion $\left(1.0 \mathrm{x} 1.0 \mu \mathrm{m}^{2}\right)$ scanned with peak forces of $0.4 \mathrm{nN}$ are shown in Figures $1 \mathrm{c}$ and $1 \mathrm{~d}$ allowing the micro-structuration of the adsorbed surfactant layer to be clearly and better evidenced. No evidence of ordered extended structures could be found on mica substrates, as shown by topographic images obtained on the same substrate region by scanning the adsorbed film over different size areas (Fig. 2). The general features remain the same both in terms of structure and of morphology of the adsorbed film from intermediate to high resolution. The similarity and reproducibility in the series of images taken at different magnifications ensure that the scanning parameters we used in the PFT-AFM mode (applied load, scanning rate, etc.) are adequate and do not damage the fragile adsorbed film.
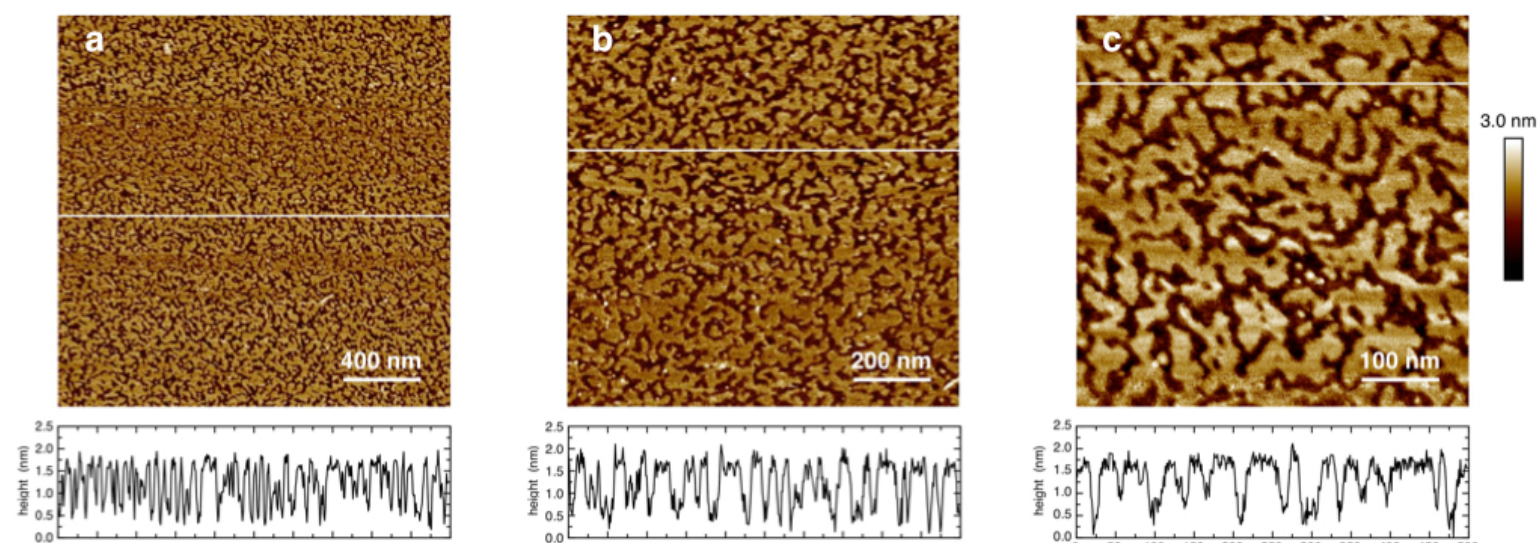

Figure 2: Height images of a $C_{16} T A B$-coated mica surface immersed in a $C_{16} T A B$ solution at $\approx 1 / 120$ cmc and equilibrated for $24 \mathrm{~h}$ at $25{ }^{\circ} \mathrm{C}$. Using the PFT-AFM mode the same spot was scanned over different size areas: (a) $2 \mu \mathrm{m} \times 2 \mu \mathrm{m}$; (b) $1 \mu \mathrm{m} \times 1 \mu \mathrm{m}$; (c) $0.5 \mu \mathrm{m} \times 0.5 \mu \mathrm{m}$ showing that the weak load applied by the tip $(0.4 \mathrm{nN})$ does not damage the structural pattern once the thermodynamic equilibrium is attained: the adsorbed film is in a quenched state. The bottom plots show the height profile along the horizontal white line for each image. They reveal bare mica areas surrounded by adsorbed $C_{16} T A B$ regions regular in thickness $(1.7-1.9 \mathrm{~nm})$, although one cannot exclude the presence of surfactant molecules lying flat down below the monolayered-like stripes. The film morphometry composed of patches is the same at any spatial scale over the whole mica surface without any longrange order.

Regarding the nature of the domains, several pieces of evidence identify them as bare mica regions left without any adsorption (although one cannot exclude the presence of isolated $\mathrm{C}_{16} \mathrm{TAB}$ molecules lying flat) and surrounded by an adsorbed film interpreted as a hydrophobic monolayer. Indeed, four arguments advocate for this conclusion. The first two remarks are related to the thickness and smoothness of the adsorbed layer. The adsorbed film appears homogeneous in height (rms roughness of less than $0.5 \mathrm{~nm}$ over distinct sample zones) with a value $(\approx 1.7 \mathrm{~nm})$ close to that previously measured by direct force measurement 
in the same solution conditions. ${ }^{45}$ As revealed by the height profile along any line across the images (Fig.1c and Fig.2), the structural features are regular in thickness. The peak-to-valley height (in the range 1.7-1.9 $\mathrm{nm}$ ) indicates that in these regions the alkyl chains are upright and tilted. Indeed, the film thickness is smaller than the length of a fully extended $\mathrm{C}_{16} \mathrm{TA}^{+}$ion $\left(\approx 2.3 \mathrm{~nm}\right.$ including the polar head thickness $\left.{ }^{54}\right)$. The small height variation suggests different tilt angles of the alkyl chains and hence different surfactant packing within the meandering monolayered-like stripes adsorbed on mica. However, the adhesion image (Fig.1d) reveals that all the regions behave in a similar manner. This third argument suggests that some flipflopped molecules with headgroups pointing toward the aqueous medium lie likely on top of the hydrophobic monolayer. Obviously, even with a low applied force as used in the PFTAFM mode, such molecules cannot be imaged individually, and the imaging technique, although advanced and less invasive compared to any other and the AM-AFM previous mode, may still deteriorate the structure. Indeed a force of $0.4 \mathrm{nN}$ is still quite considerable in the realm of soft matter, the work of this force over a vertical displacement of $0.1 \mathrm{~nm}$ is about 10 $k_{\mathrm{B}} T$. The exact magnitude of the in-plane force is not known but believed to be lower by two orders of magnitude; its work over $1 \mathrm{~nm}$ would still exceed $1 k_{\mathrm{B}} T$. Although the PFT-AFM allows the structure to be detected, which is a considerable advance, it may deform it (see also $\S$ 4-2). This likelihood of perturbing the soft sample, particularly the shape of surface aggregates at the solid-liquid interface, is probably related to their actual hydrophobic nature. Indeed, last but not least, this fourth remark lies on the macroscopic contact angle measured on the $\mathrm{C}_{16} \mathrm{TAB}$-coated mica surface. At this bulk surfactant concentration, the advancing contact angle of water is around $94^{\circ}{ }^{45}$ It is, within experimental error, the same as on surfaces prepared by Langmuir-Blodgett deposition of double-chain surfactants (for example compare with the $93^{\circ}$ value measured with dimethyldioctadecylammonium bromide ${ }^{113}$ ). In the latter case, a regular hydrophobic monolayer composed of tightly packed surfactants is formed on the mica surface, whereas in the $\mathrm{C}_{16} \mathrm{TAB}$-coated mica surface obtained by adsorption of surfactants molecules from bulk, the surface is composed of patchy layers. It is clear that contact angles of water on a hydrophobic surface on their own cannot give any insights into the microstructure of the adsorbed film.

All these four sets of remarks harmonize for claiming that the adsorbed surfactant film is micro-structured with bare mica regions surrounded by hydrophobic layered patches of surfactants that have adsorbed with their polar heads next to the surface and the hydrocarbon chains in contact with the aqueous phase. These adsorbed surfactants are not tightly packed; their alkyl chains are distributed from upright to tilted configurations. The surfactant patchy regions do not create any ordered lateral organization at long-range. On top of these hydrophobic regions are lying some flip-flopped surfactant molecules with headgroups pointing toward the aqueous medium. Similarly some isolated surfactants may lie flat on the mica regions not covered by the hydrophobic patches.

As a conclusion to this section, despite the difficulties inherent to the conventional AFM modes, the structures revealed by AFM-AM mode (Fig.1a,b) are in good agreement with the ones previously obtained in "soft contact mode" confirming the conclusive evidence of adsorbed surfactant structures at dilute bulk concentrations well below the cmc. Furthermore, the PeakForce mode has allowed the micro-structuration of the $\mathrm{C}_{16} \mathrm{TAB}$-coated mica surface to be better and confidently evidenced (Fig.1c,d and Fig.2). The PFT-AFM mode has minimized any detrimental effect and has not disrupted the patchy structures of the adsorbed layer as it images the substrate with the lowest possible force. The absence of long and lasting evolution (days), provided the thermodynamic equilibrium has been attained (see $\S 4-1)$ corroborates this conclusion. 


\section{Evolution of the morphometry of the adsorbed films as a function of the $C_{16} T A B$ concentration}

From the observations reported in the previous section, it appears that the recent PFTAFM mode overcomes most of the difficulties due to the invasive nature of the AFM imaging technique that may affect the apparent shapes and dimensions of fragile objects. Taking thus the opportunity of having a suitable tool for a direct investigation of the surfactant-coated mica structure, we present now the morphological evolution of the adsorbed film as a function of the bulk $\mathrm{C}_{16} \mathrm{TAB}$ concentration. The key to our approach is to use a delicate force which is insufficient to disrupt and displace the surfactant aggregates but has sufficient stability and proximity to discriminate surface structures.

During the course of an experiment, at a fixed location on the sample, an area of different sizes was scanned several times with the lowest possible applied peak force. In most of the cases, an area of given size was also scanned with gradually increasing peak force. The peak force threshold was varied between $150 \mathrm{pN}$ and $5 \mathrm{nN}$, so the forces used ranged over almost two orders of magnitude. The smallest force was limited by the minimum interaction required for the imaging to occur, whereas the largest force was set by the apparent disappearance of (most of) the surfactant film features from the height image. As the force is proportional to the cantilever stiffness, which was almost the same $(\approx 0.7 \mathrm{~N} / \mathrm{m})$ in our series of experiments, the force range is related to the balance between hydrophobic and electrical double layer interactions that operate in aqueous medium between the tip and the surfactantcoated mica substrate to be imaged.

While the adsorbed areas stayed attached under the minimum force imposed by the tip, the structure could be effected during imaging upon application of greater forces during repeated scanning: displacement, local destruction or sweeping away organic material was observed, especially for the mica surfaces equilibrated in the lowest $\mathrm{C}_{16} \mathrm{TAB}$ concentration solutions. Changes in the structural features but also in the physico-chemical properties of the adsorbed film can also be revealed in the so-called adhesion, dissipation and deformation images acquired simultaneously with the height images thanks to the PFT-mode. Therefore, particular attention was given to the comparison between the images sets (height, adhesion, dissipation, deformation) acquired at the lowest possible applied force and the ones scanned at larger forces. We have noted that disruption of the structural features of the fragile adsorbed film is most often irreversible and must be prevented: a new scan of the same area with a lower force does not restore the morphometry of the adsorbed surfactant film to its original state. Thus, in order to avoid clearly flawed images, the interactions between the AFM tip and the sample must be minimized: the first scan was done with the lowest possible force, i.e. with the force for which almost any features were visible in the image. After the scanning of the area was completed, we immediately increased slightly the force and continued the scanning. Verifying that positions, cross-sections, shapes, lateral sizes, and orientations of the layered aggregates, did not change upon the scanning, we considered that the set of images (height, adhesion, dissipation, deformation) is representative of the morphometry of the $\mathrm{C}_{16} \mathrm{TAB}$-coated mica in equilibrium with the given bulk surfactant concentration. As we will discuss in $\S 4-1$, this is one of the criteria for assessing the achievement of the thermodynamic equilibrium.

Such exemplary images, all scanned using the PFT-AFM mode, are shown in Figures 3 to 8 . In the series of independent experiments presented in this section, we systematically investigated surface adsorption of $\mathrm{C}_{16} \mathrm{TAB}$ on mica in surfactant aqueous solutions spanning the concentration range from $1 / 1000 \mathrm{cmc}$ to $2 \mathrm{cmc}$.

At the lowest surfactant concentrations the topographic images do not reveal any surface aggregates. However, several arguments concur on the existence of single surfactant 
molecules adsorbed on the mica substrate. The first remark lies on the observation that the mica substrate acquires a small surface roughness compared to its initial atomic smoothness when it is freshly cleaved. The estimation of the surface roughness at microscopic scale was determined by imaging different areas of $2 \times 2 \mu \mathrm{m}^{2}$ per sample using the root mean square roughness parameter $R_{\mathrm{a}}$ calculated with the AFM software. It increased up to $R_{a} \approx 0.3 \mathrm{~nm}$ in any direction indicating that the mica-water interface remains homogeneous and isotropic. The second remark is related to the nature of the interactions between the tip and the substrate. In pure water, the force-distance profile is repulsive at large separations due to the overlap of the electrical double layers but becomes attractive close to contact as van der Waals interactions dominate. Mica immersion in these low concentrated surfactant solutions immediately increases the magnitude of the attraction at short separations: the larger attraction and pull-off force indicate that a hydrophobic contribution to the total interaction has arisen. It results from the adsorption of some $\mathrm{C}_{16} \mathrm{TA}^{+}$ions. The small total roughness value $\left(R_{a} \approx 0.3\right.$ $\mathrm{nm}$ ) suggests that these adsorbed surfactant molecules are likely isolated (single molecules or assembled in very small clusters) and lie parallel to the mica substrate with their hydrophobic alkyl chains exposed to the aqueous medium. Unfortunately, the image contrast and the resolution of the PFT-AFM are not high enough to allow single adsorbed molecules to be imaged (they may also be swept away upon the scanning) and thus prevent any conformation to be revealed.

It is only above $\sim 1 / 300 \mathrm{cmc}$ that some organization of the adsorbed $\mathrm{C}_{16} \mathrm{TA}^{+}$ions is revealed by the PFT-AFM. The evidenced small spots on the images suggest that selfassembly of adsorbed surfactants has occurred. The aggregates have a lateral extension of a few nanometers, a height of less than $\approx 1.5 \mathrm{~nm}$, and are randomly distributed over the mica surface (Fig. 3). The homogeneous texture of the adhesion image with a small amplitude variation suggests that the alkyl chains of the adsorbed surfactant molecules are exposed to the aqueous medium whenever they are, either self-assembled in the aggregates or isolated in the bare mica regions. According to the concept of Gaudin and Fuerstenau, the associated monomers adsorbed to the surface with the headgroups next to the surface and the hydrocarbon chains in contact with the aqueous phase compose hemimicelles. ${ }^{14}$ The surfactant packing appears loose as the height of the aggregates is smaller than a full extended $\mathrm{C}_{16} \mathrm{TAB}$ molecular length $(\approx 2.3 \mathrm{~nm})$. This suggests that the alkyl chains may be tilted at least by an angle of $\cos ^{-1}(1.5 / 2.3) \approx 50^{\circ}$ with respect to the surface normal direction.
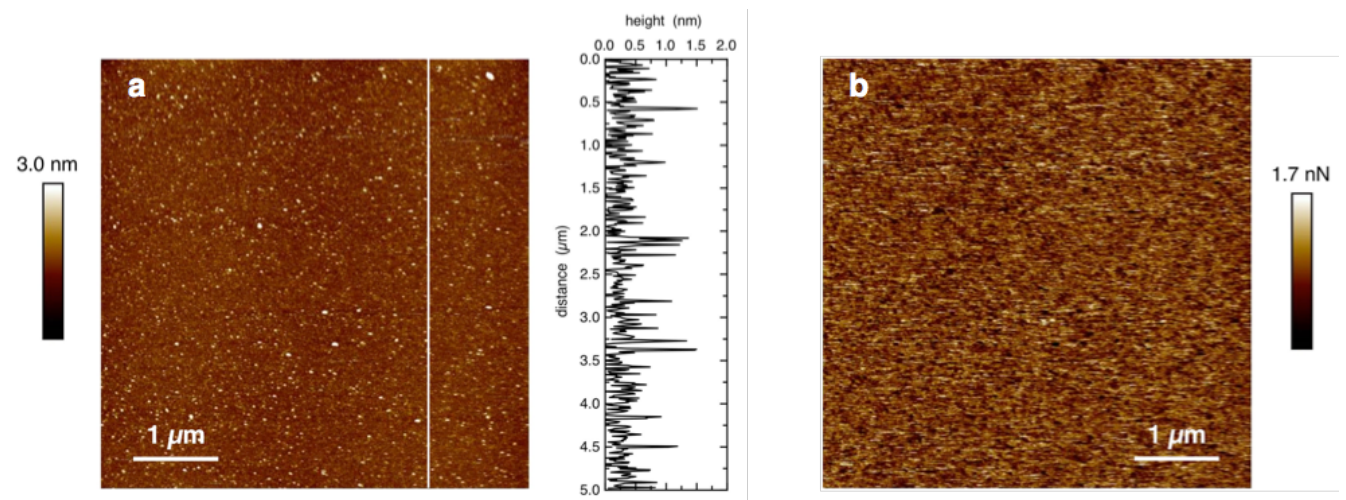

Figure 3: (a): PFT-AFM height image $(5 \mu \mathrm{m} \times 5 \mu \mathrm{m})$ of a $C_{16}$ TAB-coated mica surface immersed in a $C_{16} T A B$ solution at $1 / 165 \mathrm{cmc}$. The surface aggregates have a small lateral extension $(<10 \mathrm{~nm})$ and a height not more than $1.5 \mathrm{~nm}$ as exemplified by the height profile along the vertical white line. (b): The homogeneity in the adhesion image suggests that the physico-chemical properties are similar over the whole surfactant-coated mica surface: the $C_{16} T A^{+}$ions in the surface aggregates expose their hydrophobic alkyl chains to the aqueous medium as the surfactant molecules do when they lie flat on the mica substrate. 
The formation of distinct monolayer islands of $\mathrm{C}_{16} \mathrm{TA}^{+}$adsorbed on mica from a dilute aqueous solution is followed by further growth of the islands and nucleation of new islands as the bulk $\mathrm{C}_{16} \mathrm{TAB}$ concentration is increased. The hemimicelles extend rapidly their lateral size (Fig. 4) but remain flattened despite a slight increase in their thickness $(1.4-2.0 \mathrm{~nm})$. The increased height indicates that the alkyl chains have stood up due to a likely tighter packing of the surfactant molecules in the adsorbed aggregates (tilt angle of $\cos ^{-1}(1.7 / 2.3) \approx 42^{\circ}$; this interpretation is valid for large aggregates; for the ones with smaller lateral sizes, the edges are likely to play a non negligible role).
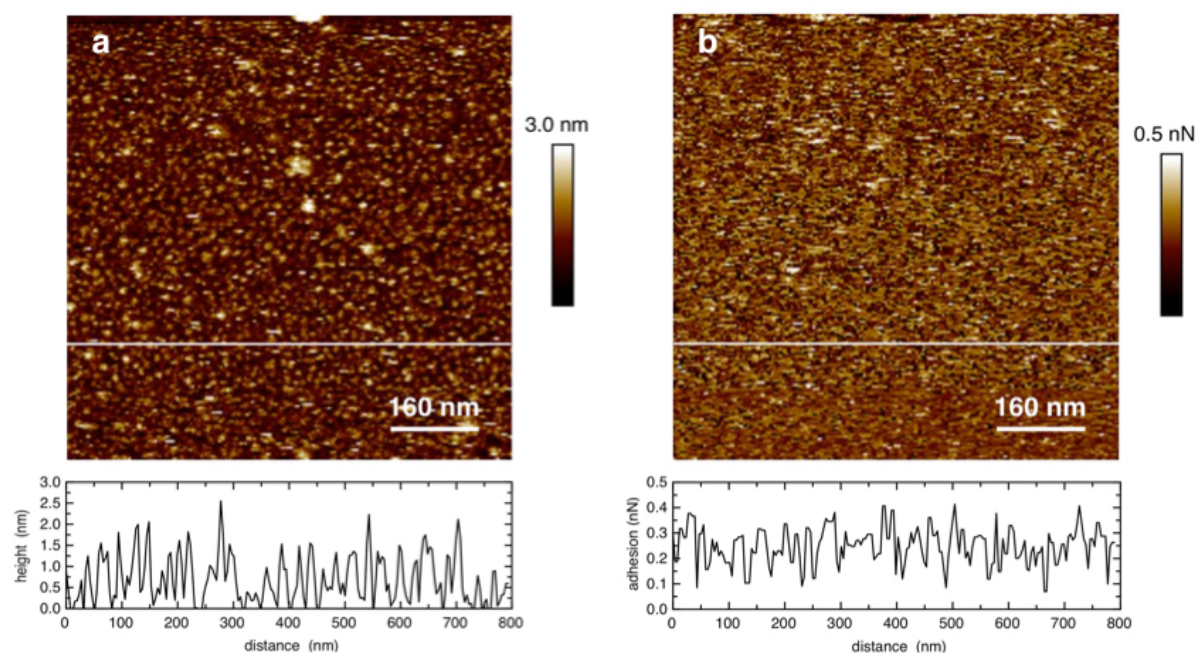

Figure 4: (a): PFT-AFM height image $(0.8 \mu \mathrm{m} \times 0.8 \mu \mathrm{m})$ of a $C_{16}$ TAB-coated mica surface immersed in a $C_{16} T A B$ solution at 1/150 cmc. Compared to Fig.3, the surface aggregates have increased their lateral extension (>10 nm) and their height slightly (range between 1.4 and $2 \mathrm{~nm}$ ) as exemplified by the height profile along the horizontal white line. (b): The adhesion image remains homogeneous over the whole surfactant-coated mica surface: the small variation in the amplitude (adhesion profile along the same section of the topograph) suggests a variation in the packing of the surfactant molecules either lying flat on the bare mica substrate or being self-assembled in the flattened hemimicelles.

Increasing the bulk $\mathrm{C}_{16} \mathrm{TAB}$ concentration leads to a new aspect of the images. At low concentrations, the adsorbed aggregates are imaged as dots that clearly are not connected to one another (Figs. 3 and 4). With a concentration increase the islands eventually begin to coalesce and form semicontiguous meandering stripes (Fig. 2). Note that this transformation occurs over a rather narrow increase of the bulk surfactant concentration. Indeed, contiguous regions covering the substrate begin to be visible at already $1 / 140 \mathrm{cmc}$ (Fig. 5). Remarkably, the stripes have a thickness similar to the height of the isolated hemimicelles, at a value around 1.7-1.9 $\mathrm{nm}$ : they can be considered as monolayered-like aggregates. In this narrow concentration range, the adsorption occurs through the growth of existing aggregates rather than through the formation of new ones. This is clearly evidenced in Figures 5a-c with the report of different image sizes. As noted previously (Figs. 1d, $3 b$ and $4 b$, the adhesion maps indicate similar conformations taken by the adsorbed surfactant molecules (Figs. 5d-f). With alkyl chains pointing towards the aqueous medium, the small variation of the adhesion encountered along the surface aggregates suggests different packing of the surfactant molecules. The denser the surface aggregate the higher the adhesion, since the hydrophobic water contact is the largest.

The height of these anisotropic domains is slightly higher than the isolated aggregates that were formed at lower concentrations, but still remains shorter than a fully extended surfactant molecule. This suggests a closer packing of the self-assembled surfactants with alkyl chains forming a tilt angle from the surface normal direction. In the concentration range 

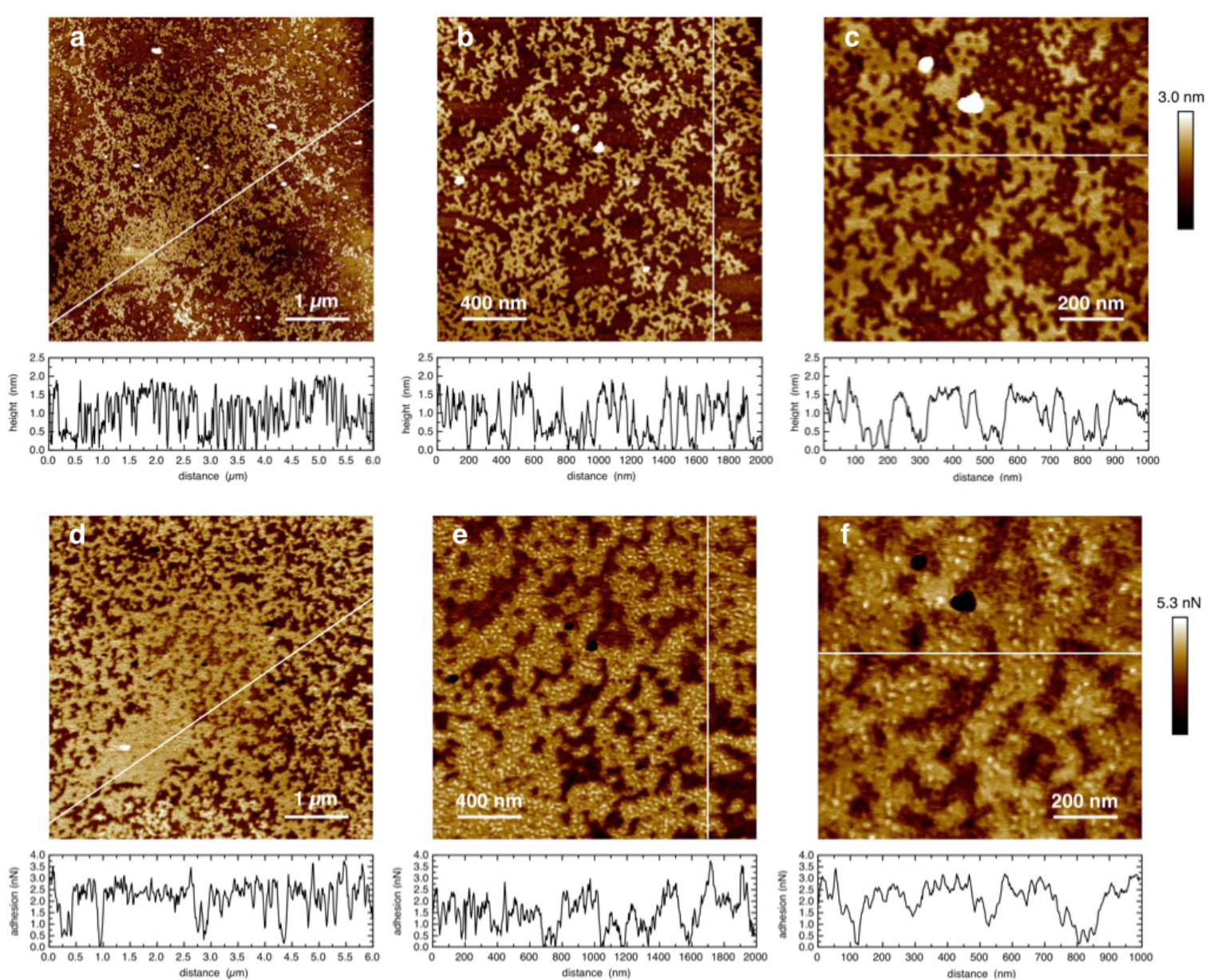

Figure 5: PFT-AFM height $(a, b, c)$ and corresponding adhesion images $(d, e, f)$ of a $C_{16}$ TAB-coated mica surface immersed in a $C_{16} T A B$ solution at $1 / 140 \mathrm{cmc}$. Scanning the same region over different sizes $(5 \mu m \times 5 \mu m(a, d) ; 2 \mu m \times 2 \mu m(b, e) ; 1 \mu m \times 1 \mu m(a, f))$ reveal the coexistence of isolated islands and contiguous meandering stripes. They all are monolayered-like aggregates as indicated by their thickness in the 1.7-1.9 $\mathrm{nm}$ range (profiles along the white lines of the topographs) smaller than a full extended $C_{16} T A B$ molecular length $(\approx 2.3 \mathrm{~nm})$. The small variation in the adhesion along the surface aggregates (profiles along the same sections) suggests different packing of the surfactant molecules exposing their hydrophobic alkyl chains at the aqueous interface.

between $1 / 130$ and $1 / 90 \mathrm{cmc}$ the morphology of the $\mathrm{C}_{16} \mathrm{TAB}$ coated-mica film is the same (Fig.1c; Fig.2; Fig.6). It has a patchy appearance with the coexistence of covered areas, not more than $2 \mathrm{~nm}$ high, and bare mica regions (with probably some isolated adsorbed surfactant molecules lying almost flat) distributed uniformly across the mica surface without the propagation of any lateral order over the substrate surface. ${ }^{71}$ The adsorbed film presents the same features as already described in details in $\S 3-1$. Only the surface coverage increases with the bulk surfactant concentration in which the immersed mica substrate is at equilibrium. At about $1 / 90 \mathrm{cmc}$, the mica substrate is almost entirely covered by a continuous homogeneous monolayer hydrophobic film (Fig. 6). Nevertheless, it is likely that some $\mathrm{C}_{16} \mathrm{TAB}$ molecules in a flip-flop configuration lie on top of it. Indeed, direct force measurements (SFA) have revealed a net positive surface charge (although small) at about this concentration. ${ }^{45}$ The fact that such top molecules (or clusters) cannot be easily imaged, even with the PFT-AFM mode, indicate that they are loosely attached onto the monolayeredlike film, as lateral forces involved in the scanning by the tip action are prone to the displacement and eventually removal of materials weakly adsorbed. 

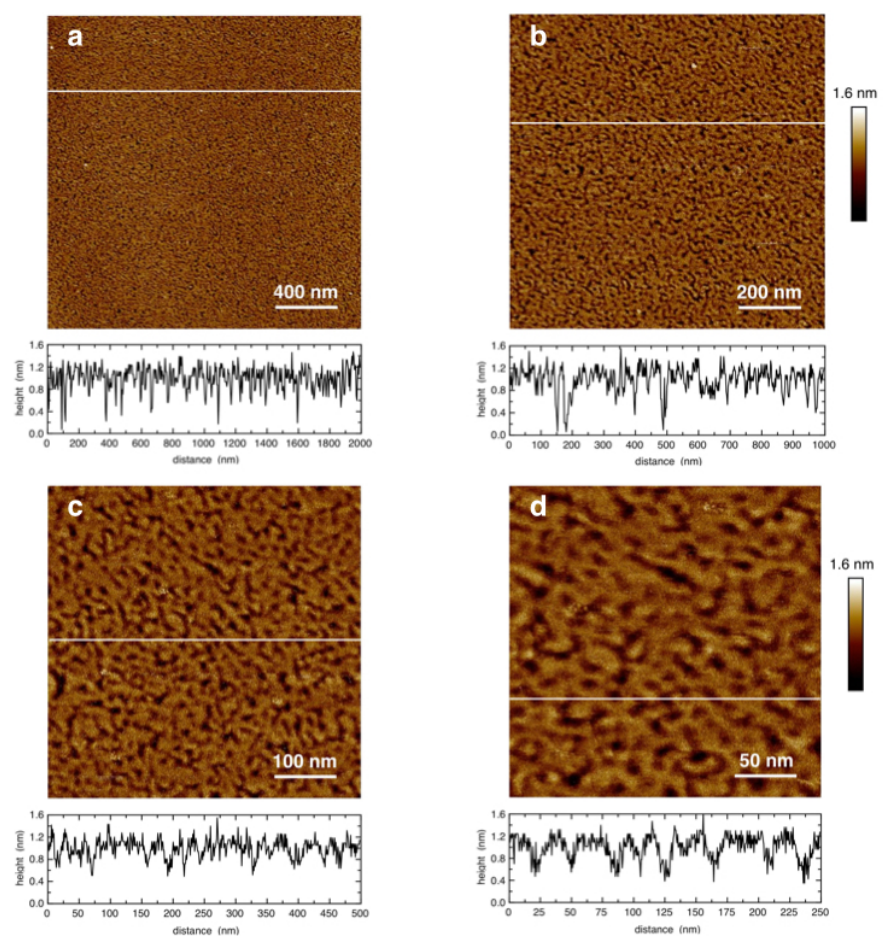

Figure 6: Height images of a $C_{16} T A B$-coated mica surface immersed in a $C_{16} T A B$ solution at $\approx 1 / 90$ cmc. Using the PFT-AFM mode the same spot was scanned over different size areas: (a) $2 \mu \mathrm{m} \times 2 \mu \mathrm{m}$; (b) $1 \mu \mathrm{m} \times 1 \mu \mathrm{m}$; (c) $0.5 \mu \mathrm{m} \times 0.5 \mu \mathrm{m}$; and (d) $0.25 \mu \mathrm{m} \times 0.25 \mu \mathrm{m}$. At this concentration, the monolayered-like film forms a connected space that covers the mica substrate almost entirely: only a few holes are still present. The bottom plots show the height profile along the horizontal white line for each image. Note that the scale in the z-direction is only relative as the zero origin is unknown (the bare mica surface could not be contacted with the tip around this region to avoid tip damage upon film penetration and scratch).

Increasing further the concentration allows the occurrence of a patched pattern to be revealed again (Fig. 7). But these patches have now a thickness larger than the previous one (monolayered-like film). Because they are higher than a full extended $\mathrm{C}_{16} \mathrm{TAB}$ molecular length they are interpreted as partial bilayered-like regions with top surfactant molecules oriented with headgroups facing toward the aqueous solution. The total thickness is in the range 3.0-3.2 $\mathrm{nm}$. This value is in very good agreement with the adsorbed film thickness in similar solution conditions as measured by SFA, ${ }^{42,45}$ by X-ray reflectometry, ${ }^{54}$ and by SFAX, combining both SFA and small angle x-ray scattering (our work recently published ${ }^{71}$ and to be published). We note that the total height is smaller than two fully extended $\mathrm{C}_{16} \mathrm{TAB}$ molecules suggesting that the surfactant molecules are either interdigitated or tilted. As it will be discussed in $\S 4-2$, the second interpretation is favored. The height distribution reveals a slight increase in the thickness of the underlying monolayer film, namely by $0.2-0.3 \mathrm{~nm}$ compared to that at lower concentrations. This suggests either a closer surfactant packing, or the presence of surfactant molecules lying on top of the hydrophobic monolayer, that can no longer be swept away by the AFM tip upon scanning, probably because of the presence of the bilayered-like surface aggregates forming a patched network.

More and more large islands of double thickness develop on top of the continuous monolayer film as the surfactant concentration continues to be increased. These partial bilayers expand their lateral size over the surface and get closer. Nevertheless, these bilayered-like surface aggregates do not touch each other. Indeed, such a configuration would be highly unfavorable because of the hydration and electrostatic repulsions between the polar 


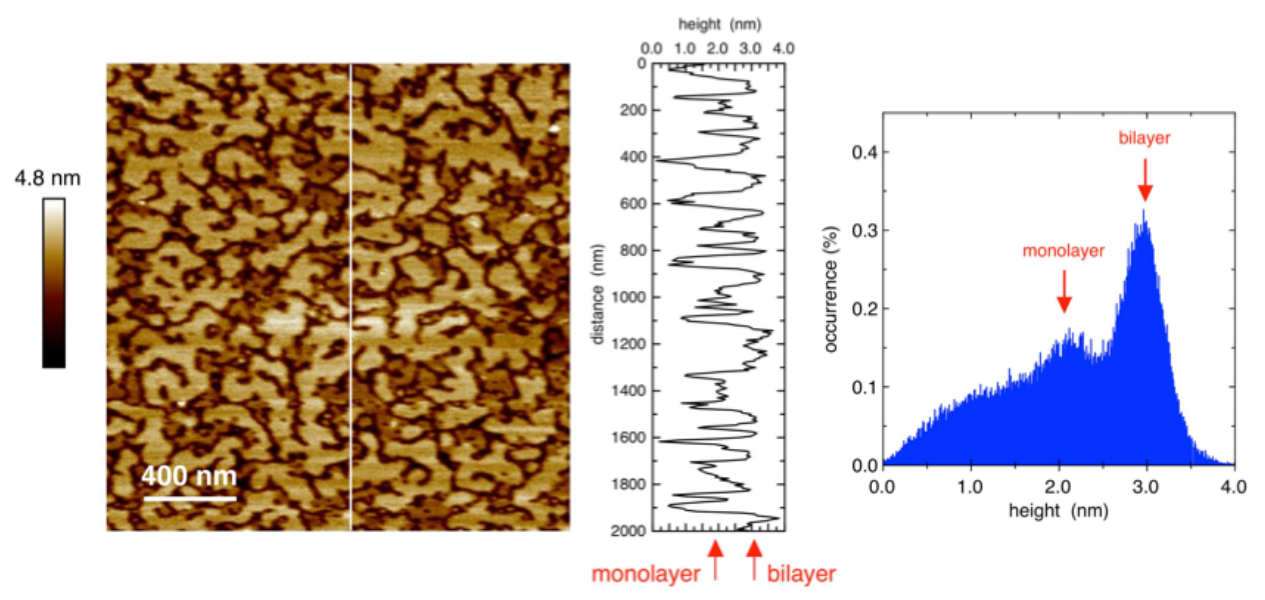

Figure 7: PFT-AFM height image of a $C_{16}$ TAB-coated mica surface immersed in a $C_{16}$ TAB solution at $\approx 1 / 70 \mathrm{cmc}$. Bilayered-like surface aggregates have formed on top of the underlying hydrophobic monolayer that covers almost entirely the mica substrate as shown by the height profile along the vertical white line and by the histogram of the height distribution.

heads of adjacent admicelles. It is much easier for the system to fuse the aggregates into larger ones. In this way the elongation of the bilayered-like surface aggregates could be driven by interaggregate interactions. Still, one has to explain why the pattern also becomes finite along the length of the aggregates. Because interaggregate forces will not produce a modulation of the stripes along their length, the effect of intra-aggregate forces must be considered instead. In other words, the line tension that relates the curvature of the interface between the amphiphiles and the aqueous medium will tend to reduce the aspect ratio of the surface aggregates shape. The balance between these forces leads to the contiguous meandering stripes as observed in Figure 7.

The fusion is not straightforward since the modulation along the length of the stripes may also be a precursor for their connections. Indeed, further growth of stripe width is prohibited by repulsion; it may then become more advantageous to accommodate the extra $\mathrm{C}_{16} \mathrm{TAB}$ by making connexions between neighboring stripes (some occurrences are already visible at the $\approx 1 / 70 \mathrm{cmc}$ concentration as illustrated by Figure 7 ). This generates a twodimensional network of interconnected bilayered-like surface aggregates. A similar situation was observed in 3D for a network of ribbons merging two-by-two sideways to transform into a continuous rhombohedral network. ${ }^{114,115}$ There the decrease in the mean curvature is accompanied by a saddle-splay geometry (negative Gaussian curvature) for the apolar/polar interface reducing the cost in energy. ${ }^{116}$ Concerning the $\mathrm{C}_{16} \mathrm{TAB}$ adsorbed film a similar mechanism can be invoked to describe the formation of a network connecting the surface bilayered-like aggregates, although a proper theory must consider not only the mean curvature and the Gaussian curvature, but also their spatial gradients.

Very rapidly the film composed of connected stripes becomes a full $2 \mathrm{D}$ closed-cell network. From $1 / 50$ to $1 / 10 \mathrm{cmc}$ the network pitch reduces progressively and concomitantly the regions exposing the monolayer film shrink. Even if at $1 / 10 \mathrm{cmc}$ the film still contains a collection of defects, it can be seen as a piece of bilayer which would be corrugated. Ultimately as the $\mathrm{C}_{16} \mathrm{TAB}$ concentration approaches the $\mathrm{cmc}$, the images are almost featureless, indicating a one-phase film. Regions exposing the monolayer film are no longer visible: the connected stripes have turned into a homogeneous flat complete bilayer covering entirely the mica substrate (Fig. 8).

The replacement of the previous patchy appearance by a continuous adsorbed film occurs at around $1 / 2 \mathrm{cmc}$. This threshold in $\mathrm{C}_{16} \mathrm{TAB}$ concentration is in very good agreement 


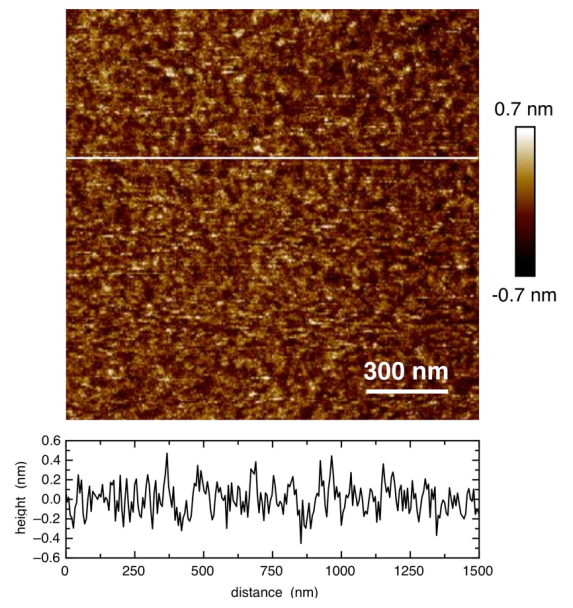

Figure 8: PFT-AFM height image of a $C_{16} T A B$-coated mica surface immersed in a $C_{16} T A B$ solution at the cmc. The adsorbed film is homogeneous, flat (root-mean-square (rms) height deviation of $0.2 \mathrm{~nm}$ ), and covers entirely the mica substrate. It is interpreted as a complete bilayer (see text). Only relative $z$-scale is given, since the zero level can be obtained only after disruption of the structure upon large applied loads. The bottom plot shows the height profile along the horizontal white line.

with previous SFA ${ }^{45}$ and X-ray reflectometry ${ }^{54}$ investigations. Note that the PFT-AFM mode is of advantage to reveal the absence of discrete structures as minimum load is applied by the scanning tip onto the coated-mica film. Obviously, we sought for direct evidence that an adsorbed film is actually present. Two arguments can advocate for. First, large applied loads favor the disruption and erosion of the adsorbed surfactant layer (not shown). Second, for the PFT-AFM images showing a uniform surface, the captured force curves reveal a long-range electrical double layer repulsion between the AFM tip and the surface whereas upon closer approach, the tip can be pushed through the adsorbed layer.

We note that the lack of any clear observable structural feature in the range of a few tenths of cmc may be the source of possible misinterpretation, where one could hypothesize that no surfactant has adsorbed on the mica substrate. Such claim is quite disingenuous and is clearly ruled out with regard to the following double argument. The nature of the tip-sample interactions is still about the same as the ones measured at lower concentrations, particularly when the presence of a strong attractive well at contact indicates the contribution of a hydrophobic interaction. And even more if taken together, the evolution of both the adsorbed film morphology and the surface coverage as a function of the bulk surfactant concentration. The more concentrated the solution is, the most the surfactant patches cover the mica substrate. It is clear that our systematic investigation of the $\mathrm{C}_{16} \mathrm{TAB}$-coated mica film morphometry along its adsorption isotherm is of advantage to discriminate between full coverage of a smooth substrate and its opposite. An AFM observation carried out at one given concentration only is limited on its own. This may explain also why in the past, beyond the complications generated by the more invasive nature of the other AFM modes used before the recent development of the PeakForce, many previous AFM reports have claimed in a erroneous way that no surfactant adsorption occurred in the range of a few tenths of cmc.

\section{Discussion}

\section{Thermodynamic equilibrium}

In order to obtain valid structural parameters of the surfactant adsorbed film from the AFM technique, it is valuable that the system under study be at equilibrium before any imaging scan is undertaken. A particularly noteworthy feature of the alkyl- 
trimethylammonium surfactants adsorption kinetics is the extremely long time required for non-micellar solutions to reach their adsorption plateaus. ${ }^{117}$ Equilibration times may vary by several orders of magnitude for different surfactant concentrations that spanned just over one order of magnitude below the cmc: thus, it was observed that $\mathrm{C}_{16} \mathrm{TAB}$ required a few minutes to attain $90 \%$ of its adsorption plateau from a solution above the cmc in contact with silica, but half an hour at $0.9 \mathrm{cmc}$, and $11 \mathrm{~h}$ (the plateau being reached after $14 \mathrm{~h}$ ) at half the $\mathrm{cmc} .{ }^{117}$ Polarized infrared attenuated reflection (IR-ATR) confirms this slow kinetics: at $\sim 1 / 16 \mathrm{cmc}$ while $50 \%$ of the equilibrium amount is adsorbed within $2 \mathrm{~h}$ of silica immersion, $80 \%$ in $10 \mathrm{~h}$, a total in excess of $30 \mathrm{~h}$ is required to reach full adsorption equilibrium. ${ }^{79}$ This observation is in agreement with the AFM report stating that the texture of the layer formed on silica in contact with a $\mathrm{C}_{16} \mathrm{TAB}$ solution at $\sim 1 / 15 \mathrm{cmc}$ could be successfully imaged after suitable time (8-16 h) only. ${ }^{64}$ Contrary to high-purity silica, the negative surface charges of clay minerals, such as muscovite and montmorillonite, are compensated by exchangeable surface cations. Nevertheless, similar observations about long equilibration times are also reported. Thus, it was observed that some time between 6 and $20 \mathrm{~h}$ after the mica is exposed to $\mathrm{C}_{16} \mathrm{TAB}$ solutions at $2 \mathrm{cmc}$, the structure evolves to flat bilayers ${ }^{33,34}$ and the flat sheet structure is retained after one month. ${ }^{33}$ Similar behaviors were observed when the surfactants posses a different counterion (such as chloride ${ }^{33}$ ), a variable chain length, and adsorb on substrates of variable surface charge density (mica, silica): for instance for $\mathrm{C}_{18} \mathrm{TAB}$ the adsorption process continues up to about $40 \mathrm{~h}$ after the silicon wafer was exposed to a $1 / 40 \mathrm{cmc}$ solution ${ }^{118}$ while on silica, even at $2 \mathrm{cmc}$, the repulsive interaction was reported to stiffen over many hours. ${ }^{119}$ Interestingly, on mica the formation of layer was suggested to proceed via growth of islands over periods that could be exacerbated up to days if the temperature was decreased below the Krafft boundary. ${ }^{60,120}$ At room temperature (but above the Krafft boundary) the direct measurement of interactions between two similar $\mathrm{C}_{16} \mathrm{TAB}$-coated mica surfaces using a Surface Force Apparatus showed that several hours of equilibration ( $<12$ hours) were sufficient for obtaining reproducible results at small fractions of the $\mathrm{cmc}$, but $24 \mathrm{~h}$ (or more) was necessary in the low concentration regime $(<1 / 100 \mathrm{cmc})$ as the surfactant aggregates were found to be not anchored sufficiently strongly to the mica and could be displaced upon applied loads. ${ }^{45}$

These long equilibration times suggest that the kinetics of adsorption is not governed only by convective-diffusion from the bulk to the surface, in series with a surface "reaction", but rather that the adsorption mechanism is reaction-limited. Such slow anchoring and surface aggregate organization may involve many complex rate-determining processes, e.g. slow diffusion of molecules at the interface, hindered penetration and/or overturn of surfactant molecules in the adsorbed film. Indeed, since $\mathrm{C}_{16} \mathrm{TAB}$ causes a net surface charge reversal at very low coverage whether it is adsorbed on mica $^{45}$ or on silica, ${ }^{72}$ virtually all the adsorbing $\mathrm{C}_{16} \mathrm{TA}^{+}$ions must diffuse against an electrostatic repulsion from the positively charged adsorbed layer. This electrostatic hindrance retards considerably the rate of diffusional (nonconvective) adsorption. In addition to these lower surfactant adsorption rates after the rapidly occurring initial adsorption phase, several mechanisms concour to a very slow occurring equilibration phase. Monte Carlo simulations underline the importance of exchange competition between surfactants and inorganic cations of the substrate (potassium ions for the muscovite mica), gradual release of inorganic surface cations paired with initially coadsorbed surfactant counterions out of the aggregate regions, and rearrangement of surfactant aggregates. ${ }^{56}$

Concerning the anchoring of surfactant molecules on a substrate one can note that the extensive hydrogen bond of water molecules on muscovite surface plays a role of an obstacle for surfactant adsorption. Indeed, Infra-Red, ${ }^{121}$ X-ray reflectivity ${ }^{122}$ and Monte Carlo simulations $^{123}$ all agree to reveal the existence of an adsorbed layer of water molecules bound 
intimately to the ditrigonal cavities in the mica surface and surmounted by a network of hydration water molecules into which $\mathrm{K}^{+}$counterions are interposed consistently with hydrogen bonding to the surface $\mathrm{O}$. With a first layer of water molecules that are tightly bound to the surface the bonding network is more structured and rigid than that of bulk water. ${ }^{121}$ This makes its penetration and disruption more difficult and the time-scale for attaining a solid and definitive anchoring of a cationic surfactant molecule is slowed down. This is especially exacerbated with quaternary ammonium head groups $\left(\mathrm{R}-\mathrm{N}\left(\mathrm{CH}_{3}\right)_{3}{ }^{+}\right)$ compared to primary ammonium head groups $\left(\mathrm{R}_{-} \mathrm{NH}_{3}{ }^{+}\right)$. While both head groups bond ionically to the surface, $\mathrm{NH}_{3}$ can form additional $\mathrm{N}-\mathrm{H}$... $\mathrm{O}-\mathrm{Si}$ hydrogen bonds to the surface. ${ }^{124-126}$ In contrast, because $\mathrm{N}\left(\mathrm{CH}_{3}\right)_{3}$ is larger in size the O...H and N...O distances would become too large to allow hydrogen bonds to bridge oxygen atoms of the surface. ${ }^{124,125}$ According to Monte Carlo simulation the $\mathrm{C}_{16} \mathrm{TA}^{+}$ions penetrate the first adsorbed water layer, desorb the doubly hydrogen-bonded water molecules from the mineral surface, and ultimately adsorb above ditrigonal cavities with the methyl group closest to the surface positioned directly above the cavity center (see Fig.5 of ref. 56), the adsorption site occupied by water molecules in the case of unmodified muscovite. Another effect of the large head group size is that the electric charge for $\mathrm{N}$ is partly spread over the three adjacent $\mathrm{CH}_{3}$ groups and the $\mathrm{CH}_{2}$ group of the alkyl chain. As pointed out by molecular dynamics simulations several consequences arise. First, the interaction energy with the surface is smaller (in absolute value) by about a factor 3 compared with $\mathrm{R}_{-} \mathrm{NH}_{3}{ }^{+}$surfactants: the adsorption energy between $\mathrm{C}_{16} \mathrm{TA}^{+}$surfactants and the mica surface is computed to $\sim-40 \mathrm{kcal} / \mathrm{mole} .{ }^{125} \mathrm{Second}$, the absence of hydrogen bonds facilitates more frequent rearrangements across cavities in the mica surface. This leads to a flexible orientation of the $\mathrm{R}-\mathrm{N}\left(\mathrm{CH}_{3}\right)_{3}{ }^{+}$head group on the surface and greater lateral mobility with a higher rate of diffusion as the surfactant molecules exhibit more hopping on the surface. ${ }^{124}$ Finally the large steric hindrance of the head groups also slows down the surfactant self-assembly on the surface. Thus, Neivandt et al. observed by IRATR that at low surface coverages the $\mathrm{C}_{16} \mathrm{TAB}$ molecule is randomly orientated during initial adsorption; as the adsorption process progresses and the adsorbed amount increases with time, the average orientation of the surfactant molecule toward the normal rises so as to maximize packing density. ${ }^{79}$

These slow structural changes are consistent with the X-ray Photoelectron Spectroscopy (XPS) results of Chen et al., which showed a slow increase in the density of $\mathrm{C}_{16} \mathrm{TA}^{+}$molecules at the mica surface. ${ }^{55}$ In the model proposed by these authors some surfactants may adsorb initially either as a $\mathrm{C}_{16} \mathrm{TA}^{+}$cation (exchanging with a $\mathrm{K}^{+}$ion) but also as a dipole with its $\mathrm{Br}^{-}$counterion (exchanging with water molecules). Over a period of time, the trapped counterion for the surfactant and the counterion for the solid diffuse as an ion pair out of the headgroup region through the (hydrophobic) layer and exit the film to leave the surfactant directly bound to the solid. Ultimately, after long equilibration times as revealed by XPS, no bromine atoms ${ }^{120}$ or only low adsorption densities of surfactant counterions ${ }^{55}$ are left. In terms of structure, the release of interfacial counterions from the surface region and the replacement of a dipole-dipole bond by a much stronger Coulombic ionic interaction result in an increasingly stronger binding of the $\mathrm{C}_{16} \mathrm{TA}^{+}$ions in the inner layer to mica with a concomitant rearrangement of the hydration region around the trimethylammonium headgroups. ${ }^{56}$ In terms of time scale, to leave the muscovite surface, $\mathrm{K}^{+}$ions have to overcome the Coulomb repulsion barrier of the $\mathrm{C}_{16} \mathrm{TA}^{+}$headgroups facing the muscovite surface. Then, they have to diffuse across the hydrophobic and low dielectric parts of the mono/bilayered like film in construction (as the $\mathrm{Br}^{-}$must do as well), and eventually overcome a second Coulomb repulsion barrier of the $\mathrm{C}_{16} \mathrm{TA}^{+}$headgroups facing the adsorbed aggregate - solution interface. These energy barriers impede the release of $\mathrm{K}^{+}$ions from the interfacial muscovite region. Both the slow departure of small cations and anions from the 
surface film and the slow adsorption of surfactant cations induce the surface film to reach equilibrium slowly. Thus the final equilibrium state is achieved after times of hours up to days as observed experimentally.

Taking advantage of the molecular dynamics simulation results as they present not only the atomic level insight into the microstructure of the adsorption system but also provide the dynamic and thermodynamic information for the adsorption process, we now discuss the importance of the route to be chosen experimentally so as to be certain that the AFM technique really investigates the adsorbed $\mathrm{C}_{16} \mathrm{TAB}$ film at the thermodynamic equilibrium. As a result, for every surfactant concentration of the bulk solution in contact with the mica surface, not only the morphometry, the size and the shape of the adsorbed aggregates can be inferred (as described in $\S 3$ ), but also a surface coverage can be determined (see $\S 4-2$ ). However, such a determination in situ is useful and reliable only if one avoids the complications similar to those encountered in solution depletion experiments for adsorption isotherms measurement where solute concentration and $\mathrm{pH}$ may change considerably during the adsorption process. In principle, the small surface area of the substrate $\left(\sim 0.5 \mathrm{~cm}^{2}\right)$ ensures that the adsorption takes place under constant conditions in the bulk solution. Nevertheless, the small volume of the AFM liquid cell $(\sim 0.3 \mathrm{~mL})$ implies a not so negligible surface area to solution volume ratio. Therefore upon surfactant adsorption, together with a decrease in $\mathrm{pH}$ ( since $\mathrm{H}^{+}$is a potential determining ion for mica ${ }^{25,127}$ ) the composition in bulk solution can be depleted, especially in the low surfactant range investigated in this study. To overcome these difficulties different routes can be proposed.

One general procedure is to feed the solution that has been depleted in surfactant content by gradually replacing it with a fresh one at the desired bulk concentration. As slow equilibration times for the adsorption are expected (of the order of one day), one may flush the AFM fluid cell on a regular periodicity basis (a few hours). Another attractive procedure is to use a slow continuous flow that will be maintained over extended periods by means of a syringe pump. Beyond the need for 2-4 hours solution replacement there are several advantages of using a syringe driver. As precise and reliable administration of very small fluid deliveries (in the micro, nano, and pico volumetric flow rate ranges) can be achieved, the continuous infusion avoids peaks and troughs by maintaining constant bulk surfactant levels in the AFM fluid cell over the course of the long adsorption process. Nevertheless, despite these benefits with operating and setting up syringe drivers, we noted some adverse effects for the specific study reported here. Actually, the negative limitation arises from the very principle of such a procedure since a syringe driver is a dynamic administration system, which means that when the solution flows, hydrodynamic disorders can lead to poor or lack of adsorption, as the kinematic boundary can induce unstable interfaces. This predicament is especially exacerbated for the quaternary alkyl ammonium surfactants: onto the muscovite mica, a strong anchoring of the $\mathrm{N}\left(\mathrm{CH}_{3}\right)_{3}{ }^{+}$head groups - large in size with a high lateral mobility - requires some rearrangements of their hydration shells which is a slow process as discussed above through the literature observations. As a consequence of the positive displacement provided by the syringe pump that forces fluid through the AFM fluid cell, loose attached surfactant molecules can be scraped off from the mica and swept away before they get time to anchor more strongly on the substrate. From our observations, it is clear that syringe drivers do not achieve optimal building of the surfactant film on the mica substrate, even at very small volumetric flow rates. Figure 9 illustrates this conclusion when we investigated the use of such a procedure in the low $\mathrm{C}_{16} \mathrm{TAB}$ concentration range, well below the cmc. The syringe pump was operated open loop, specifying a particular rate at which the syringe is actuated, directly controlling the flow rate through the AFM fluid cell. The evidenced small spots on the images suggest that self-assembly of adsorbed surfactants has occurred. At $\approx 1 / 165 \mathrm{cmc}$, most of the aggregates have a lateral extension of a few to tens of 
nanometers, a height of $\approx 1.5 \mathrm{~nm}$, and are randomly distributed over the mica surface. However, none of the characteristic patterned micro-structuration of the adsorbed film is revealed at higher concentrations (Fig. $9 \mathrm{~b}$ to be compared with Figs. 1,2,5,6). Even with slow flow rates, most of the surfactant molecules appear to be swept away during the equilibration process: the few white spots seen in Fig. 9b that have larger lateral and higher dimensions (up to $13 \mathrm{~nm}$ ) suggest that there is accumulation of $\mathrm{C}_{16} \mathrm{TAB}$ molecules around some already well anchored surfactant molecules that play the role of attachment sites of the aggregates. Nevertheless, those are too high and disorganized to resemble the mono/bi-layered-like fragments encountered in Figures 1, 2, 5, 6, 7. Here, the continuous flow exchange prevents distinct, densely packed, monolayer islands of $\mathrm{C}_{16} \mathrm{TA}^{+}$to build up on mica from a dilute aqueous surfactant solution.

From this observation, namely that the adsorbed surfactant molecules with alkyl chains oriented toward the aqueous solution act as nucleation centers for surface aggregates formed through chain-chain association in the later stage of adsorption, two suggestions can be drawn. First, the competitive character of the adsorption must not be neglected in model considerations and in setting experimental procedures. The individual surfactant adsorption is a first stage that supplies the nuclei to the later surface aggregation. It occurs through ion exchange and rearrangement of the hydrated shells. The presence of the hydrophobic moiety around the surfactant ions has an influence on the mechanism of adsorption. Already at the end of individual adsorption of surfactant ions at low surface coverages, hydrophobic interactions are sufficiently strong to provoke the formation of clusters and surface aggregates. The latter stage of the adsorption process cannot occur at all in the case of adsorption ascribed to electrostatic interactions only. ${ }^{128}$ Secondly, our observation suggests a slow kinetics of island nucleation and growth in agreement with previous reports. ${ }^{63}$ For instance, the onset of $\mathrm{C}_{18} \mathrm{TA}^{+}$island formation was observed to occur after several hours of exposure of the mica substrate to the assembly solution, before which time there is no significant ordering of the alkyl chains observed. ${ }^{60,63,129}$ Once the critical nucleus has reached a size large enough, the significant barrier to island nucleation is overcome, and the islands can further grow thanks to the (slow) surface transport of surfactant molecules not anchored strongly yet. Eventually, these islands may ultimately coalesce and form a semi-contiguous mono/bi-layered-like fragments at the mica surface. Because the island growth is contingent on charge migration and rearrangement of the hydration regions together with some structural changes, the micro-structuration formation involves long transient times due to large structural and energetic changes which are propagating from the nucleation sites in all directions through the vicinity. In conclusion, we decided not to use a continuous flow of bulk surfactant solution as it appears to be an inappropriate procedure for insuring the formation of reliable $\mathrm{C}_{16} \mathrm{TAB}$-coated mica films in situ within the small AFM volume cell.
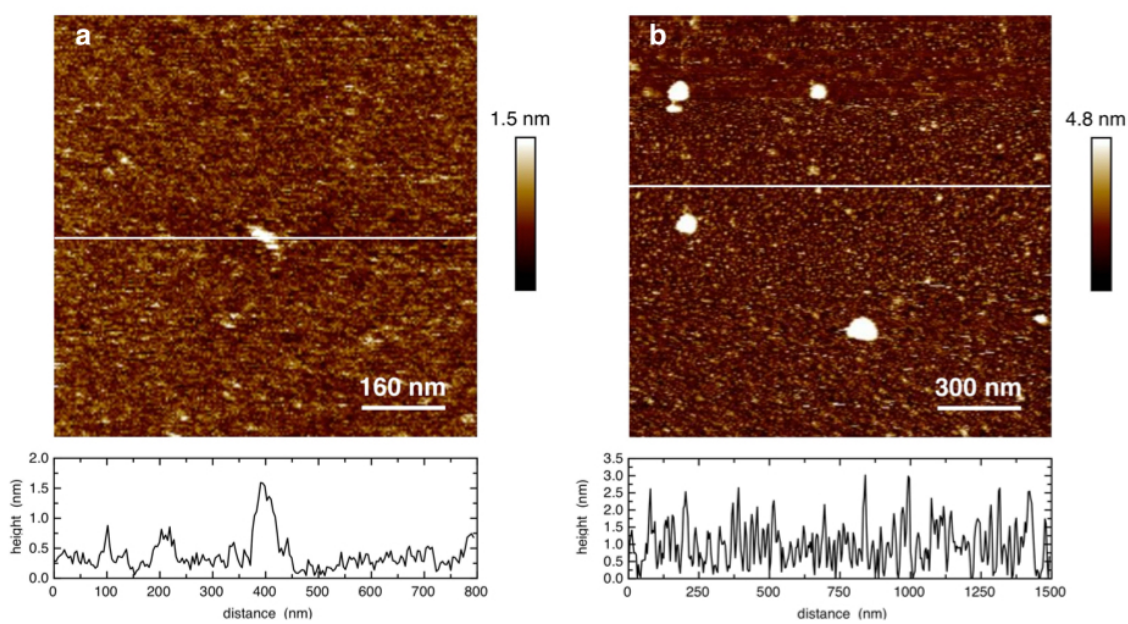
Figure 9: PFT-AFM height images of $C_{16} T A B$-coated mica surfaces in equilibrium with bulk surfactant aqueous solutions at $1 / 165 \mathrm{cmc}$ (a) and $1 / 117 \mathrm{cmc}$ (b). These images were recorded after a $\sim 20 \mathrm{~h}$ continuous flow rate of $\sim 25 \mathrm{pL} / \mathrm{s}$ that has allowed the $\sim 0.3 \mathrm{~mL}$ volume of the AFM fluid cell to be fully replaced every 3-4 hours at each respective bulk surfactant concentration. The flow favors the formation of larger aggregates (compare Fig.9a with Fig. 3 at the same concentration of 1/165 cmc). This effect is exacerbated at higher concentrations (Fig.9b) with accumulation of surfactant molecules around anchoring points on the mica substrate. These regions (white spots) are large ( $100 \mathrm{~nm})$ and quite high ( 6-8 nm) and even very high (although more rarely) up to $13 \mathrm{~nm}$ (for the white spot lying in the bottom middle of the image). Note that the continuous flow has impeded any micro-structuration of the adsorbed film at 1/117 cmc (compare with Figs. 1,2,5,6).

This is why we rather preferred to resort to the classical and convenient procedure as described in $\S 2$ where the mica substrates in the AFM fluid cell are directly equilibrated in contact with the surfactant solution, that is regenerated by gentle flushing a couple of times over the $24 \mathrm{~h}$ period. This convenient method gives a better control of the conditions during the adsorption than the continuous flushing method. It was also checked that the results obtained at a given concentration were the same independently of sequential addition of $\mathrm{C}_{16} \mathrm{TAB}$ solutions. Thus for some experiments, the $\mathrm{C}_{16} \mathrm{TAB}$ concentration in the AFM fluid cell was changed by subsequent additions of higher concentration solutions. The volume added in each step was equivalent to 6 times the total cell volume to ensure that the AFM fluid cell is well-flushed. At each concentration step the $\mathrm{C}_{16} \mathrm{TAB}$ was equilibrated with the mica surface prior to a more concentrated solution being added. Similar structures and morphometry of the adsorbed films were observed, whether the freshly cleaved mica substrates were in contact (and equilibrated) directly with the surfactant solution at the desired concentration, or after having been equilibrated at lower concentrations before increasing concentrations through incremental steps. Finally, a more general experiment was performed at $\mathrm{C}_{16} \mathrm{TAB}$ concentrations around $1 / 100 \mathrm{cmc}$ to check if the characteristic patterned microstructuration (Figs. 1,2,6) would still evolve for equilibration times longer than the $24 \mathrm{~h}$ period used in the present work $(\S 3)$, even after reinjection of the solution. Our observations carried out after more than two days of equilibration did not reveal any variations of the adsorbed film both qualitatively and quantitatively compared to its state after 1 day: the morphometry, the structural organization and the surface coverage remained the same with no evidence of higher features with increased immersion. In other words, at the thermodynamic equilibrium the adsorbed film can be considered as a quenched system.

A final remark concerns the role of the temperature in $\mathrm{C}_{16} \mathrm{TAB}$ adsorption on mica. All the data presented in this work were obtained at $25^{\circ} \mathrm{C}$, above the Krafft boundary (the crystal solubility boundary of surfactant ${ }^{107}$ ), with a rigorous protocol to produce well defined and reproducible morphologies of the adsorbed films. To this end, we would reiterate the need for such a suitably approach towards any deviations that can be potentially detrimental to surfactant adsorption from solution. Minor changes may give rise to substantial consequences. Thus, we emphasize the importance to get an explicit control of thermal history. This includes maintaining that temperature during solution preparation (see $\S 2$ ), while avoiding any temperature fluctuations around the Krafft boundary to prevent surfactant precipitation and coexistence with $\mathrm{C}_{16} \mathrm{TAB}$ crystal hydrates. Therein lies the profound significance of a constant temperature upon solution injection into the AFM fluid cell and during the long equilibration process. Any temperature drop below the Krafft boundary, even small and temporarily short, may affect the formation of the patterns as those reported in $\S 3$. Thus, we observed a variety of film morphologies, most often inhomogeneous, with spurious variations in their thicknesses (presence of spikes). Once such features and domains were formed (results not shown), most often they could not be annealed even after long equilibration times above the Krafft temperature. These may be attributed to precipitation of crystal hydrates that 
may eventually adsorb onto the mica surface. Indeed, the Krafft discontinuity is a eutectic phase transition; the dilute phase is a liquid phase and the concentrated phase a crystal phase. ${ }^{107}$ As the surfactant crystal phase does not vanish but persists to temperatures above the Krafft boundary, it is suggested that these domains exist as long-lived metastable phases (possibly forming a variety of polymorphic structures). The important structural variations induced below the Krafft boundary suggest many changes in the physical properties of the surfactant solution. It was already noted that the coexistence with crystal hydrates has a profound effect on phase changes - and its occurrence is not unique to aqueous cationic surfactants (see examples given for many surfactant systems in ref. 107 and in refs. 130,131 for sodium dodecyl sulfate). Here we see it has also a considerable impact on the adsorption process. The effects concern not only the electrostatics and hydration interactions, but also the surface (line) tension set along the surface aggregates due to the presence of alkyl chains that are no longer in a liquid-like state. As a consequence, it appears that the Krafft boundary (temperature) is a very important parameter for aqueous $\mathrm{C}_{16} \mathrm{TAB}$ solutions adsorbing on a substrate.

\section{Surface coverage}

Being certain that the adsorbed $\mathrm{C}_{16} \mathrm{TAB}$ films on the mica surfaces are at the thermodynamic equilibrium, not only the morphometry, the size and the shape of the surface aggregates can be inferred from the AFM images collected for every surfactant concentration of the bulk solution in contact with the mica surface (as described in $\S 3$ ), but also a surface coverage can be determined. Such information is valid provided meaningful data are obtained by the AFM technique. Indeed, while high vertical resolution images of the different structures can be obtained, caution must be exercised in their interpretation, as the topography generated can be a (strong) function of imaging parameters especially when one investigates fragile samples. Indeed, as already pointed out, the mechanical action of the AFM tip is prone to disrupt the structural features of soft samples and hence the $\mathrm{C}_{16} \mathrm{TAB}-\mathrm{mica}$ coated films. In addition, high-resolution imaging of such films adsorbed to substrates becomes a very challenging task for AFM: the finite dimensions of the AFM probe which determine the extension of the surface area interacting with the tip do not allow detailed structural features of the surface aggregates to be imaged at the molecular level. These remarks set the framework and the limitations of the present investigation. The inferred structural parameters can be understood as valid through the following remarks and cautions.

The morphometry of the $\mathrm{C}_{16} \mathrm{TAB}$-mica coated films was investigated by means of the most advanced and recent PFT-AFM mode. By maintaining constant the force applied by the AFM tip upon scanning the substrate, the likehood of perturbing fragile samples is minimized, since the better the applied force can be controlled (i.e., the lower it can be maintained) the less chance of sample and/or tip damage. In the present investigation the normal force applied was typically about $0.4 \mathrm{nN}$. This magnitude is well below the force required to break apart a surface structure. Indeed, experimental data show that the force needed to disintegrate a $\mathrm{C}_{16} \mathrm{TAB}$ micelle structure adsorbed on mica is $\sim 2 \mathrm{nN}{ }^{132}$ in good agreement with molecular dynamics. ${ }^{133}$ Moreover this value can be considered as a lower bound, especially for adsorbed aggregates formed on mica after long times as in our present investigation. At the thermodynamic equilibrium these surface aggregates are well anchored onto the charged substrate because both the electrostatic interactions with the polar heads and the lateral interactions between surfactant tails are optimized. Therefore, a higher density of cohesive surfactant molecules in the interaction area of the AFM tip would result in a stronger elastic response. This remark underlines the importance of extent of surfactant cohesion in the adsorption process and the benefit of investigating the morphometry of the adsorbed film at 
the thermodynamic equilibrium. As an illustration pointed out by Rabinovich et al. it is likely that adsorbed cylindrical micelles are more cohesive than the spherical structures given the same participating molecules: as a result more energy applied by the normal load is required in order to destroy these structures. ${ }^{132}$ In the present investigation, different nanoscale structures cause variations in surfactant density, which may affect the derived mechanical properties. Nevertheless, for the flatten mono/bi-layered-like aggregates as observed in the present report we can consider that the weak applied normal force used in the PeakForce mode $(\sim 0.4 \mathrm{nN})$ is likely to have little disruption in the morphology of the adsorbed structures.

This first remark points out the benefit of the PFT-AFM that allows the overall structure to be detected, which is a considerable advance. However, it needs also to be tempered because of the limitations induced by the finite size of the AFM tip. This drawback inherent to any AFM imaging mode induces several consequences. The tip-sample convolution effect - the interaction between the tip and surface features being imaged, is one of the main causes of AFM artifacts owing to the characteristic geometry and finite sharpness of tips. ${ }^{134-136}$ Contrary to structures with steep sidewalls, where numerous artifacts, such as "shadow", 134 "arc-like", ${ }^{136}$ or even "additional material", ${ }^{134}$ have been noted, the boundaries of the adsorbed domains investigated in the present study are rather soft, rounded, and not sharp. It is likely that the side edges of the aggregates abutting the aqueous medium do not expose upright hydrocarbon chains only: there are also surfactant molecules with their polar heads facing water in order to decrease the apolar extent of the lateral interface. With a thickness of the adsorbed domains in the range 1.5 to $4 \mathrm{~nm}$, i.e. smaller or of the order of the tip radius $\left(r_{\text {tip }}=2-10 \mathrm{~nm}\right)$, such detailed features are clearly impossible to be evidenced due to insufficient lateral resolution. But the finite size of the AFM tip adds to the measured length of any imaged object in the plane of image, whereas height measurements are generally unaffected by the tip convolution with the sample. The topographic image can be described as the morphological dilation of the surface by the tip. The dilation broadens peaks and bumps while holes and pits shrink. Thus, accurate quantitative analysis becomes limited with the tendency of overestimating the lateral dimensions of the imaged objects due to tip convolution. ${ }^{23}$ Nevertheless, some estimate of the convolution effect can be derived as follows:

All along the adsorption isotherm ( $\S 3)$, the morphometry of the adsorbed films is that of mono/bi-layered-like domains, that are globally flat even if their thicknesses may vary from one to another fragment. The dependence of the convolution error for a rectangular motif (width $w$; height, $h$ ) with different aspect ratios, $h / w$, and a given $r_{\text {tip }}$ has been discussed by Canet-Ferrer et al. . ${ }^{137}$ Since the radius of curvature of the tip used in PFT-AFM is usually reported to be in the range $2-10 \mathrm{~nm}$ and around a statistical value of about $4 \mathrm{~nm}$, the film thickness of mono/bi-layered-like domains, $h$, which is of the order of $2-4 \mathrm{~nm}$, is smaller than the tip round $\left(h \leq r_{\text {tip }}\right)$. As a consequence, the difference between the measured width, $w_{\text {meas, }}$ and the real width, $w$, of the stripes is $w_{\text {meas }}-w=2 r_{\text {tip }} \cos \left\{\operatorname{Arcsin}\left[\left(r_{\text {tip }}-h\right) / r_{\text {tip }}\right]\right\}$. This leads to a convolution error of $1.7 r_{\text {tip }} \approx 7 \mathrm{~nm}$ for mono-layered-like fragments, increasing slightly to $2 r_{\text {tip }} \approx 8 \mathrm{~nm}$ for bilayered-like domains of double thickness, but being not negligible for low objects (convolution error of $0.9 r_{\text {tip }} \approx 3.5 \mathrm{~nm}$ for $h=0.1 r_{\text {tip }} \approx 0.4 \mathrm{~nm}$ ). Hence, as the height of the object under study is reduced, the influence of $r_{\text {tip }}$ increases. Although the convolution error is not completely independent from the motif shape and its aspect ratio, Canet-Ferrer et al. ${ }^{137}$ showed by comparing simulations performed on 2D rectangular and elliptical objects that in most the cases, the shape dependence is generally minor or negligible (the convolution error for rectangular objects is aspect ratio independent at constant $h$ ). Thus, comparable convolution errors are obtained (slightly below $2 r_{\text {tip }}$ ) for objects as different as rectangles and circumferences allowing the experimental error to be 
estimated without knowing the object shape in detail.

Note that this estimate uses the assumption that interacting but not interpenetrating bodies are tangent at their contact point. For soft matter samples this is not necessarily the case, and also the tip surface can be altered by "contamination" (material transfer), wear or damage. The situations in which the tip would suffer wear between successive scans are not discussed here. Indeed, they were immediately abandoned experimentally because changes in tip geometry (blunter tip, double tip, etc.) will generate inconsistencies. However, there may be also some variations along the image scan as the amount of contamination via take up of debris by the tip during imaging may vary: material transfer may appear, accumulate or conversely disappear during the scan at any time that is at any sample location. The situation gets complex as the deformation of the sample during tip imaging can be reversible or not, with a dependence of many parameters, including probe shape on imaging force, magnitude of the applied force, scan speed, and sample compliance. All these effects can be rendered minimum thanks to the PFT-AFM mode, but still, the mixing between sample morphology and mechanical behavior cannot be fully avoided when imaging fragile sample features are comparable in size to the tip, thereby misleading the observations that molecular assemblies have taken on conformations that do not actually exist. Alleviating the problem is not easy and one often resorts to guesswork in image interpretation. Apart from the ideal (but inaccessible) situation when, at each location, information about the tip shape and the sample surface are known, that is the true shape is estimated and the deformation of the sample is known, putting an outer bound is nevertheless useful for any quantitative measurements and metrology purposes, such as determinations of surface coverage, lateral correlation lengths, etc.

In that respect, Table 1 summarizes our guide for assessing a quantitative statement of the uncertainty to such measured values. The various sources of uncertainties for a variable, irrespective of their provenance and type (A or B), ${ }^{138}$ are compounded using the root-sumsquare combination: $u_{\text {tot }}=\sqrt{\sum_{i} u_{i}^{2}}$ where $u_{i}=u\left(x_{i}\right)$ is the estimated standard deviation associated with the variable $x_{i}$. The surface coverage evolution as a function of the bulk surfactant concentration at which we arrived in Fig. 10 is the result of calculations involving several inputs. Thus, the process of determining the overall uncertainty consists first in determining the extent to which various factors affect the uncertainty of the inputs and then how sensitive the final result is to these input uncertainties. Because the vertical resolution is determined by the AFM instrument at a sub-nanometer level, allowing the thickness of the surface aggregates to be inferred with great accuracy, we have focused on the uncertainties in the lateral dimensions measurements only. The uncertainties due to the individual factors are refereed to as "components of uncertainty": an overview of the analysis is given in Table 1. We found three significant uncertainty components:

(i) Instrument-sample repeatability: With each tip, we recorded the topography of the sample several times. When the data are collected nominally at the same position, the image profiles may be slightly different, although the repeatability was found to be very good. Indeed, as explained in $\S 4-1$, the samples are scanned only after the thermodynamic equilibrium is reached: at this ultimate stage, the adsorbed films can be considered as in a quenched state. If any differences between the results exist, these are usually due to displacement of loose adsorbed objects upon scanning, material transfer and local disruption of the fragile surfactant film. For every bulk surfactant concentration, the images were also recorded at different spots and over different scan sizes. The differences in the distributions of the lateral sizes of the adsorbed objects (isolated spots, stripes, patches) are a measure of the uncertainty in the surface coverage calculation as a function of position along the substrate.

(ii) Uncertainty for the threshold choice: The surface coverage characterization 
requires a choice of the threshold value to discriminate between the adsorbed film and the background. When the objects appear isolated, the uncertainty is estimated by statistical means, from multiple measurements and calculation of the standard deviation. This uncertainty is of "type A" according to the nomenclature set by the Bureau International des Poids et Mesures (BIPM) and the International Organization for Standardization (ISO). ${ }^{138}$ Conversely, when the film morphometry is composed of stripes and patches that may be contiguous, some uncertainty is associated with the threshold choice, as is to be expected. The determination requires a judgment concerning the highest threshold beyond which the area is no longer changing significantly. Different choices would lead to slightly different computed coated areas. The magnitude of differences between these profiles is a measure of the uncertainty associated with the choice of threshold. Because it depends on scientific judgments based on previous measurement data, experience with the behavior and properties of relevant procedures in image analysis (see $\S 2$ ), this uncertainty is of "type B". ${ }^{138}$

(iii) Uncertainty due to tip convolution: Taking advantage of comparable convolution errors independent of the knowledge of the object shape in detail (see above), a range of $\approx 3-8 \mathrm{~nm}$ for the convolution error was considered according to the height of the adsorbed surface aggregate considered (Table 1).

It is clear from Table 1 that the tip convolution effect induces the dominant contribution to the combined standard uncertainty, $u_{\text {tot }}$, with, however, sometimes significant additional contributions from uncertainties in the effects of the threshold choice. Uncertainty arising from possible variations in the repeatability was small in comparison. This remarks underlines the importance to monitor the film morphometry once the thermodynamic equilibrium is attained.

\begin{tabular}{|c|c|c|c|c|}
\hline $\begin{array}{c}\text { Surface aggregate } \\
\text { height }(\mathrm{nm})\end{array}$ & Uncertainty component & Type & $\begin{array}{c}\text { Typical value } \\
u_{i}(\mathrm{~nm})\end{array}$ & $\begin{array}{c}\text { Total uncertainty } \\
u_{\text {tot }}(\mathrm{nm})\end{array}$ \\
\hline \multirow{3}{*}{$0.3-1$} & Instrument - sample repeatability & $\mathrm{A}$ & 2 & \multirow{2}{*}{$3.5-5.5$} \\
\cline { 2 - 4 } & Image threshold & $\mathrm{B}$ & 1 & \\
\cline { 2 - 4 } & Tip convolution & $\mathrm{A}$ & $3-5$ & \multirow{2}{*}{$7-9$} \\
\hline \multirow{3}{*}{$1-2$} & Instrument - sample repeatability & $\mathrm{A}$ & 2 & \\
\cline { 2 - 4 } & Image threshold & $\mathrm{B}$ & 5 & \multirow{2}{*}{$7.5-8.5$} \\
\cline { 2 - 4 } & Tip convolution & $\mathrm{A}$ & $5-7$ & \\
\hline \multirow{3}{*}{$2-5$} & Instrument - sample repeatability & $\mathrm{A}$ & 2 & \\
\cline { 2 - 4 } & Image threshold & $\mathrm{B}$ & 2 & \\
\cline { 2 - 4 } & Tip convolution & $\mathrm{A}$ & $7-8$ & \\
\hline
\end{tabular}

Table 1: Components of uncertainty for the lateral size determination of the surface aggregates. The combined standard uncertainty, $u_{\text {tot }}$ representing the estimated standard deviation of the result, is calculated $\left(u_{t o t}=\sqrt{\sum_{i} u_{i}^{2}}\right)$ from the estimated standard deviation of each component independent of their type. ${ }^{138}$ Its value depends on the statistical distribution of the heights of the surface aggregates composed of adsorbed surfactants that have self-assembled to form small isolated objects, elongated stripes, or patchy patterns.

Figure 10 presents the surface coverage inferred from the images $(\S 3)$ as a function of the surfactant concentration, or chemical potential, of $\mathrm{C}_{16} \mathrm{TAB}$ in the bathing solution. The bar errors reflect the reasonable limits set by the calculations summarized in Table 1 and using the height histogram of the surface aggregates of a given image. The sequence of the characteristic features of the film morphometry as described in $\S 3$ is now reflected through the surface coverage profile. However, a general caution must be underlined. As already discussed, the nonvanishing size of the AFM tip prevents a lateral resolution sufficiently high 
to reveal small objects, such as isolated surfactant molecules that could lie flat on the bare parts of the mica substrate. Thus, using AFM alone we cannot be certain whether the lower regions of the images (areas between the islands or patches) correspond to the bare mica surface, or if these regions are covered by a thin layer of $\mathrm{C}_{16} \mathrm{TAB}$ molecules. In the former scenario, the mono/bi-layered like coverage derived from the AFM images would be an accurate representation of the total surface coverage. However, in the latter case, the coverage estimate will be lower than the true total coverage. This comment also applies to the lowest concentration range, where it is difficult to infer values for any occupied areas. Nevertheless, the adhesive behavior observed in the force-distance profile is the signature of the presence of some adsorbed surfactants: the mica substrate has not been left bare but the surface occupied by these molecules is difficult to evaluate.

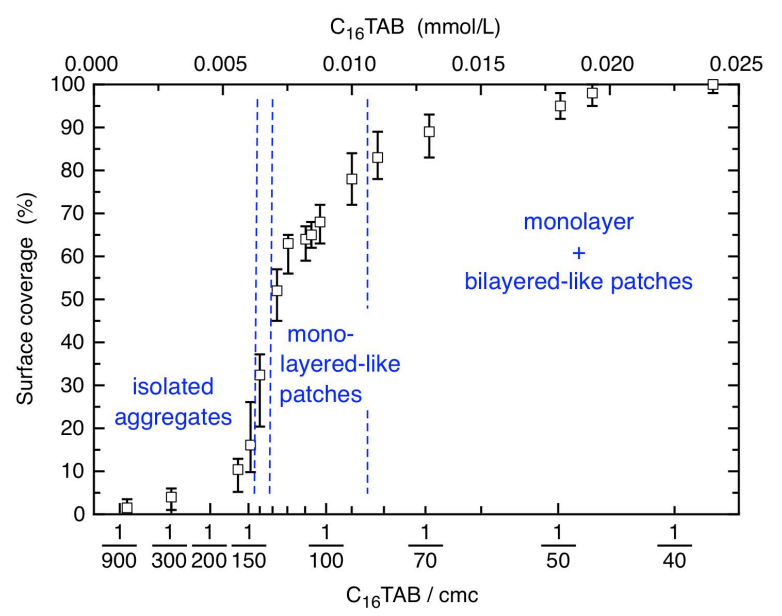

Figure 10: Surface coverage of the mica substrate as a function of the bulk $C_{16} T A B$ surfactant concentration. The surface area of the adsorbed objects as revealed by the PFT-AFM mode (isolated spots, stripes, patchy pattern) is calculated through image processing (see § 2) and the uncertainty (error bars) calculated using Table 1. As the concentration is increased, the morphometry of the adsorbed film on mica evolves in the following sequence: 1) isolated aggregates, 2) coexistence of the latter with monolayered-like patches, 3) network of connected monolayered-like patches, 4) complete monolayer, 5) bilayered-like patches on top of the underlying monolayer, and ultimately 6) complete bilayer (forming at around $1 / 2 \mathrm{cmc}$ ) covering the entire mica substrate.

\section{Conclusion}

Taking advantage of its less invasive nature compared to any other AFM modes, the PFT-AFM mode enabled high resolution topographical images to be obtained for the cationic $\mathrm{C}_{16} \mathrm{TAB}$ surfactant adsorbed on oppositely charged mica surface all along its adsorption isotherm for concentrations below the $\mathrm{cmc}$ (as low as $1 / 1000 \mathrm{cmc}$ ) and above (up to $2 \mathrm{cmc}$ ). At $25^{\circ} \mathrm{C}$ above the Krafft boundary, the evolutions of the dimensions, shapes, heights, etc., of the surface aggregates at the solid-liquid interface as well as the patterns that the adsorbed aggregates form, were obtained after long equilibration to insure the thermodynamic equilibrium. In short, for the high-charge-density mica surface, the film morphology evolves as follows. At very low concentrations the $\mathrm{C}_{16} \mathrm{TAB}$ surfactants adsorb as isolated molecules before forming small clusters and then mono/bi-layered-like meandering stripes at higher concentrations. In increasing the concentration the surfactant patches grow in lateral size, expanding and progressively filling the mica regions that were left almost bare. As the cmc is approached a homogeneous and complete bilayer covers the whole substrate ultimately. 
None of these observations would have been possible if the experimental framework for AFM investigation was not fulfilled twofold. First the use of the recent and most advanced PFT-AFM mode, allowing substrates to be imaged with the lowest possible applied force, is less invasive and minimizes interference from the AFM probe that may alter the film morphology and may cause transient structures at the surface. Secondly, perturbations of the surfactant-coated mica structure are also minimized once the thermodynamic equilibrium is reached. To fulfill this goal, a suitable and rigorous protocol with two main experimental cautions was developed: Not only long equilibration times have been allowed before any imaging scan is undertaken, but also the structural organization of the surface aggregates has not been perturbed over the course of the long adsorption process when they form and build up. This twofold remark leads also to another conclusion. The interaction of the surfactant with the oppositely charged surface is determined primarily by the head groups; the rearrangement of the hydration regions leads to stronger bonds and increases the kinetic barrier to desorption. Coupled with the hydrophobic interaction between the alkyl chains of neighboring surfactant molecules, the ultimate adsorbed aggregates get a cohesive energy sufficiently large to make any desorption process less favorable, since it would imply a collective process. At the thermodynamic equilibrium the adsorbed films behave as a quenched system as we have shown, and the film morphologies as observed by PFT-AFM are fully reproducible. Remarkably, these are in full agreement with the observations by other techniques, including other long-time limit AFM studies when available. As our experimental results reconcile with the conclusions inferred not only by the other techniques but also by the numerical simulations, our description of the adsorbed films appears to be representative of the actual situation where a cationic surfactant solution is in presence of an oppositely charged substrate.

The fact that equilibration is slow does not mean that the equilibrium adsorption state is of no consequence. On the contrary, it is perhaps even more important to independently determine the equilibrium state in systems which equilibrate slowly than in those which equilibrate rapidly. When equilibration is rapid, then kinetic factors are inconsequential. When equilibration is slow, then time and the historical treatment of the system are important in determining its state, and the equilibrium phase and adsorption state - toward which the system is headed - is unobvious as we have shown. When this situation exists a clear, independent determination of the equilibrium situation is especially important. Having focused attention on this aspect in our report does not factor into the analysis consideration of these factors which actually determine the film morphology. In filling the literature void and the lack of AFM data in the low surfactant concentration regime, one will better address the question of the hydrophilic/hydrophobic balance of a charged substrate in contact with surfactant aqueous solutions leading to the formation of patchy patterns. Within this framework, the following articles in our series will propose an analysis of the structure of the mono/bi-layered like domains or subunits that arrange on the substrate through lateral correlation lengths. This mandatory prerequisite to any modeling is crucial because it may enable one not only to understand the characteristics of adsorbed amphiphilic molecular structures at the solid-aqueous interface and how the physicochemical properties of the substrate are modified once it is coated, but also to predict the behavior in various systems, to get insights into the mechanism of the observed long-range attraction between macroscopic hydrophobic substrates, ${ }^{71,74,113,139}$ and to avoid it in those situations when the complete wetting of surfaces by water is essential.

\section{Acknowledgment}

We are grateful to Albert Johner for helpful comments and fruitful discussions. 


\section{References}

(1) Rosen, M. J. Surfactants and Interfacial Phenomena, $3^{\text {rd }}$ ed.; John Wiley \& Sons, Inc.; Hoboken, N.J., 2004, ch.3, pp. 128.

(2) Tadros, T. F. An introduction to surfactants, edited by W. de Gruyter GmbH, Berlin, Boston, 2014.

(3) Bakx, A.; Timmerman, A.; Frens, G. The flow of concentrated surfactant solutions through narrow capillaries. Colloid Polym. Sci. 2000, 278 (5), 418-424.

(4) Eastoe, J.; Hollamby, M. J.; Hudson, L. Recent advances in nanoparticle synthesis with reversed micelles. Adv. Colloid Interface Sci. 2006, 128-130, 5-15.

(5) Bakshi, M. S. How surfactants control crystal growth of nanomaterials. Cryst. Growth. Des. 2016, 16 (2), 1104-1133.

(6) Heinz, H.; Pramanik, C.; Heinz, O.; Ding, Y. F.; Mishra, R. K.; Marchon, D.; Flatt, R. J.; Estrela-Lopis, I.; Llop, J.; Moya, S.; Ziolo, R. F. Nanoparticle decoration with surfactants: Molecular interactions, assembly, and applications. Surf. Sci. Rep. 2017, 72, $1-58$.

(7) Mao, X. H.; Jiang, R.; Xiao, W.; Yu, J. G. Use of surfactants for the remediation of contaminated soils: A review. J. Hazard. Mater. 2015, 285, 419-435.

(8) Trellu, C.; Mousset, E.; Pechaud, Y.; Huguenot, D.; van Hullebusch, E. D.; Esposito, G.; Oturan, M. A. Removal of hydrophobic organic pollutants from soil washing/flushing solutions: A critical review. J. Hazard. Mater. 2016, 306, 149-174.

(9) Biresaw, G. (Ed.); Mittal, K. L. (Ed.), Surfactants in Tribology, vol. 5., Boca Raton: CRC Press, 2018.

(10) Fuerstenau, D. W.; Jameson, G.; Yoon, R.-H. Froth flotation: A century of innovation, Society for Mining, Mettallurgy, and Exploration, Inc. (SME), Littleton, Colorado (U.S.A.), 2007.

(11) Kamal, M. S. A review of Gemini surfactants: Potential application in enhanced oil recovery. J. Surfactants Deterg. 2016, 19, 223-236.

(12) Lee, H. J.; McAuley, A.; Schilke, K. F.; McGuire, J. Molecular origins of surfactantmediated stabilization of protein drugs. Adv. Drug Delivery Rev. 2011, 63, 1160-1171.

(13) Ward, R. N.; Davies, P. B.; Bain, C. D. Orientation of surfactants adsorbed on a hydrophobic surface. J. Phys. Chem. 1993, 97, 7141-7143.

(14) Gaudin, A. M.; Fuerstenau, D. W. Streaming potential studies. Quartz flotation with cationic collectors. Trans. AIME (American Institute of Mining, Metallurgical, and Petroleum Engineers) 1955, 202, 958-962.

(15) Scamehorn, J. F.; Schechter, R. S.; Wade, W. H. Adsorption of surfactants on mineral oxide surfaces from aqueous solutions I: Isomerically pure anionic surfactants. $J$. Colloid Interface Sci. 1982, 85, 463-478.

(16) Harwell, J. H.; Hoskins, J. C.; Schechter, R. S.; Wade, W. H. Pseudophase separation model for surfactant adsorption: Isomerically pure surfactants. Langmuir 1985, 1, 251262.

(17) Yeskie, M. A.; Harwell, J. H. On the structure of aggregates of adsorbed surfactants: The surface charge density at the hemimicelle/admicelle transition. J. Phys. Chem. 1988, 92, 2346-2352.

(18) Zhang, R.; Somasundaran, P. Advances in adsorption of surfactants and their mixtures at solid/solution interfaces. Adv. Colloid Interface Sci. 2006, 123-126, 213-229.

(19) Briscoe, W. H. Aqueous boundary lubrication: Molecular mechanisms, design strategy, and terra incognita. Current opinion in Colloid Interface Sci. 2017, 27, 1-8. 
(20) Heinz, H.; Ramezani-Dakhel, H. Simulations of inorganic bioorganic interfaces to discover new materials: insights, comparisons to experiment, challenges, and opportunities. Chem. Soc. Rev. 2016, 45 (2), 412-448.

(21) Atkins, R.; Craig, V. S. J.; Wanless, E. J.; Biggs, S. Mechanism of cationic surfactant adsorption at the solid-aqueous interface. Adv. Colloid Interface Sci. 2003, 103, 219304.

(22) Paria, S.; Khilar, K. C. A review on experimental studies of surfactant adsorption at the hydrophilic solid-water interface. Adv. Colloid Interface Sci. 2004, 110, 75-95.

(23) Garcia, R.; Pérez, R. Dynamic atomic force microscopy methods. Surf. Sci. Rep. 2002, 47, 197-301.

(24) Scales, P. J.; Grieser, F.; Healy, T. W. Electrokinetics of the muscovite mica-aqueous solution interface. Langmuir 1990, 6, 582-589.

(25) Shubin, V. E.; Kékicheff, P. Electrical double layer structure revisited via a surface force apparatus: Mica interfaces in lithium nitrate solutions. J. Colloid Interface Sci. 1993, $155,108-123$.

(26) Adamczyk, Z., Bratek-Sticki, A., Zeliszewska, P., Wasilewska, M., Mechanisms of fibrinogen adsorption at solid substrates. Curr. Top. Medic. Chem. Chem. 2014, 14, 702-729.

(27) de Poel, W.; Pintea, S.; Drnec, J.; Carla, F.; Felici, R.; Mulder, P. Elemans, J. A. A. W.; van Enckervort, W. J. P.; Rowan, A. E.; Vlieg, E. Muscovite mica: Flatter than a pancake, Surf. Sci. 2014, 619, 19-24.

(28) Manne, S.; Gaub, H. E. Molecular organization of surfactants at solid-liquid interfaces. Science 1995, 270, 1480-1482.

(29) Israelachvili, J. N.; Adams, G. E. Measurement of forces between two mica surfaces in aqueous electrolyte solutions in range 0-100 nm. J. Chem. Soc. Faraday Trans. I 1978, 74, 975-1001.

(30) Hanson, P. I.; Roth, R.; Morisaki, H.; Jahn, R.; Heuser, J. E. Structure and conformational changes in NSF and its membrane receptor complexes visualized by quick-freeze/deepetch electron microscopy. Cell 1997, 90, 523-535.

(31) Ducker, W. A.; Wanless, E. J. Surface-aggregate shape transformation. Langmuir 1996, 12, 5915-5920.

(32) Sharma, B. G.; Basu, S.; Sharma, M. M. Characterization of adsorbed ionic surfactants on a mica substrate, Langmuir 1996, 12, 6506-6512.

(33) Lamont, R. E.; Ducker, W. A. Surface-induced transformations for surfactant aggregates. J. Am. Chem. Soc. 1998, 120, 7602-7607.

(34) Ducker, W. A., Wanless, E. J. Adsorption of hexaecyltrimethylammonium bromide to mica: Nanometer-scale study of binding-site competition effects. Langmuir 1999, 15, 160-168.

(35) Patrick, H. N.; Warr, G. G.; Manne, S.; Aksay, I. A. Surface micellization patterns of quaternary ammonium surfactants on mica. Langmuir 1999, 15, 1685-1692.

(36) Grosse, I.; Estel, K. Thin surfactant layers at the solid interface. Colloid Polym. Sci. 2000, 278, 1000-1006.

(37) Teschke, O.; Ceotto, G.; de Souza, E. F. Imaging of soft structures: dependence of contrast in atomic force microscopy images on the force applied by the tip. J. Vac. Sci. Technol. $B$ 2000, 18, 1144-1150.

(38) Blom, A.; Duval, F. P.; Kovacs, L.; Warr, G. G.; Almgren, M.; Kadi, M.; Zana, R. Direct visualization of mesh structures at solid/solution interfaces by Atomic Force Microscopy. Langmuir 2004, 20, 1291-1297.

(39) Blom, A., Warr, G. G. Structure and Composition of cationic-nonionic surfactant mixed adsorbed layers on mica. Langmuir 2006, 22, 6787-6795. 
(40) Cao, M.; Wang, X.-L. Direct observation and distinction of the inner/outer layers of surfactant bilayer formed at the solid/solution Interface. J. Dispers. Sci. Technol. 2010, 31, 38-43.

(41) Pashley, R. M.; Israelachvili, J. N. A comparison of surface forces and interfacial properties of mica in purified surfactant solutions. Colloids Surf. 1981, 2, 169-187.

(42) Israelachvili, J. N.; Pashley, R. M. Measurement of the hydrophobic interaction between two hydrophobic surfaces in aqueous electrolyte solutions. J. Colloid Interface Sci. 1984, 98 (2), 500-514.

(43) Pashley, R. M.; Ninham, B. W. Double-layer forces in ionic micellar solutions. J. Phys. Chem. 1987, 91, 2902-2904.

(44) Pashley, R. M.; McGuiggan, P. M.; Horn, R. G.; Ninham, B. W. Forces between bilayers of cetyltrimethylammonium bromide in micellar solutions. J. Colloid Interface Sci. 1988, 126, 569-578.

(45) Kékicheff, P.; Christenson, H. K.; Ninham, B. W. Adsorption of cetyltrimethylammonium bromide to mica surfaces below the critical micellar concentration. Colloids Surf. 1989, 40 (1-2), 31-41.

(46) Helm, C. A.; Isrealachvili, J. N.; McGuiggan, P. M. Molecular mechanisms and forces involved in the adhesion and fusion of amphiphilic bilayers. Science 1989, 246, 919922.

(47) Richetti, P.; Kékicheff, P. Direct measurement of depletion and structural forces in a micellar system. Phys. Rev. Lett. 1992, 68, 1951-54.

(48) Kékicheff, P.; Richetti, P. Effect of electrolyte on the depletion and structural forces in a micellar system. Progr. Colloid Polym. Sci. 1992, 88, 8-17.

(49) Kékicheff, P.; Richetti, P. Direct measurement of interactions in supermolecular fluids and liquid crystals. Pure \& Appl. Chem. 1992, 64, 1603-1609.

(50) Kékicheff, P.; Nallet, F.; Richetti, P. Measurement of depletion interaction in semi-dilute solutions of worm-like surfactant aggregates. J. Phys. II France 1994, 4, 735-741 (1994).

(51) Kékicheff, P.; Spalla, O. Refractive index of thin aqueous films confined between two hydrophobic surfaces. Langmuir 1994, 10, 1584-1591.

(52) Griffin, L. R.; Browning, K. L.; Truscott, C. L.; Clifton; L. A.; Clarke, S. M. Complete bilayer adsorption of $\mathrm{C}_{16} \mathrm{TAB}$ on the surface of mica using neutron reflection. J. Phys. Chem. B 2015, 119, 6457-6461.

(53) Briscoe, W. H.; Speranza, F.; Li, P.; Konovalov, O.; Bouchenoire, L.; van Stam, J.; Klein, J.; Jacobs, R. M. J.; Thomas, R. K. Synchrotron XRR study of soft nanofilms at the mica-water interface. Soft Matter 2012, 8, 5055-5068.

(54) Speranza, F.; Pilkington, G. A.; Dane, T. G.; Cresswell, P. T.; Li, P.; Jacobs, R. M. J.; Arnold, T.; Bouchenoire, L.; Thomas, R. K.; Briscoe, W. H. Quiescent bilayers at the mica-water interface. Soft Matter 2013, 9, 7028-7041.

(55) Chen, Y. L.; Chen, S.; Frank, C.; Israelachvili, J. Molecular mechanisms and kinetics during the self-assembly of surfactant layers. J. Colloid Interface Sci. 1992, 153, 244265.

(56) Klebow, B.; Meleshyn, A. Aggregation of alkytrimethylammonium ions at the cleaved muscovite mica-water interface: A Monte Carlo study. Langmuir 2011, 27, 1296812976.

(57) Zana, R.; Yiv, S.; Strazielle, C.; Lianos, P. Effect of alcohol on the properties of micellar systems. I. Critical micellization concentration, micelle molecular weight and ionization degree, and solubility of alcohols in micellar solutions. J. Colloid Interface Sci. 1981, 80, 208-223. 
(58) Van Os, N. M.; Haak, J. R.; Haak, L. A. M. Physicochemical Properties of Selected Anionic, Cationic, and Nonionic Surfactants; Elsevier: New York, 1993.

(59) Nishimura, S.; Scales, P. J.; Biggs, S. R.; Healy, T. W. AFM studies of amine surfactant hemimicelle structures at the mica-water interface. Colloids Surf. 1995, 103, 209-298.

(60) Hayes, W. A.; Schwartz, D. K. Two-stage growth of octadecyltrimethylammonium bromide monolayers at mica from aqueous solution below the upoint. Langmuir 1998, 14, 5913-5917.

(61) Fujii, M.; Li, B.; Fukada, K.; Kato, T.; Seimiya, T. Heterogeneous growth and selfrepairing processes of two-dimensional molecular aggregates of adsorbed octadecyltrimethylammonium bromide at cleaved mica/aqueous solution interface as observed by in situ Atomic Force Microscopy. Langmuir 1999, 15, 3689-3692.

(62) Fujii, M.; Li, B.; Fukada, K.; Kato, T.; Seimiya, T. Two-dimensional arrangements of adsorbed alkylammonium halides on cleaved mica surface. Langmuir 2001, 17, 11381142.

(63) Mellott, J. M.; Schwartz, D. K. Supercritical self-assembled monolayer growth. J. Am. Chem. Soc. 2004, 126, 9369-9373.

(64) Fleming, B. D.; Biggs, S.; Wanless, E. J. Slow organization of cationic surfactant adsorbed to silica from solutions far below the CMC. J. Phys. Chem. B 2001, 105, 9537-9540.

(65) Manne, S.; Cleveland, J. P.; Gaub, H. E.; Stucky, G. D.; Hansma, P. K. Direct visualization of surfactant hemimicelles by force microscopy of the electrical double layer. Langmuir 1994, 10, 4409-4413.

(66) Rennie, A. R.; Lee, E. M.; Simister, E. A.; Thomas, R. K. Structure of a cationic surfactant layer at the silica-water interface. Langmuir 1990, 6, 1031-1034.

(67) Fragneto, G.; Thomas, R. K.; Rennie, A. R.; Penfold, J. Neutron reflection from hexadecyltrimethylammonium bromide adsorbed on smooth and rough silicon surfaces. Langmuir 1996, 12, 6036-6043.

(68) Penfold, J.; Staples, E. J.; Tucker, I.; Thompson, L. J. Adsorption of mixed cationic and non ionic surfactants at the hydrophilic silicon surface from aqueous solution: Studied by specular neutron reflection. Langmuir 1997, 13, 6638-6643.

(69) Penfold, J.; Tucker, I.; Petkov, J.; Thomas, R. K. Surfactant adsorption onto cellulose surfaces. Langmuir 2007, 23, 8357-8364.

(70) McDermott, D. C.; McCarney, J.; Thomas, R. K.; Rennie, A. R. Study of an adsorbed layer of hexadecyltrimethylammonium bromide using the technique of neutron reflection. $J$. Colloid Interface Sci. 1994, 162, 304-310.

(71) Kékicheff, P.; Iss, J.; Fontaine, P.; Johner, A. Direct Measurement of Lateral Correlations under Controlled Nanoconfinement. Phys. Rev. Lett. 2018, 120 (11), 118001.

(72) Parker, J. L.; Yaminsky, V. V.; Claesson, P. M. Surface forces between glass surfaces in cetyltrimethylammonium bromide solutions. J. Phys. Chem. B 1993, 97, 7706-7710.

(73) Rutland, M. W.; Parker, J. L., Surface forces between silica surfaces in cationic surfactant solutions: Adsorption and bilayer formation at normal and high pH. Langmuir 1994, 10, 1110-1121.

(74) Kékicheff, P.; Spalla, O. Long-range Electrostatic Attraction between Similar, Chargeneutral Walls. Phys. Rev. Lett. 1995, 75 (9), 1851-1854.

(75) Wong, K.; Cabane, B.; Duplessix, R.; Somasundaran, P. Aggregation of silica using cationic surfactants: A neutron-scattering study. Langmuir 1989, 5, 1346-1350.

(76) Gomez-Grana, S.; Hubert, F.; Testard, F.; Guerrero-Martinez, A.; Grillo, I.; Liz-Marzan, L. M.; Spalla, O. Surfactant (bi)layers on gold nanorods. Langmuir 2011, 28, 1453-1459.

(77) Whitby, C. P.; Scales, P. J.; Grieser, F.; Healy, T. W.; Nishimura, S.; Tateyama, H. The adsorption of dodecyltrimethylammonium bromide on mica in aqueous solution studied 
by X-ray diffraction and Atomic Force Microscopy. J. Colloid Interface Sci. 2001, 235, 350-357.

(78) Kung, K.-H. S.; Hayes, K. F. Fourier-Transform infrared spectroscopic study of the adsorption of cetyltrimethylammonium bromide and cetylpyridinium chloride on silica. Langmuir 1993, 9, 263-267.

(79) Neivandt, D. J.; Gee, M. L.; Hair, M. L.; Tripp, C. P. Polarized Infrared Attenuated Total Reflection for the in situ determination of the orientation of surfactant adsorbed at the solid/solution interface. J. Phys. Chem. B 1998, 102, 5107-5114.

(80) Ninness, B. J.; Bousfield, D. W.; Tripp, C. P. The importance of adsorbed cationic surfactant structure in dictating the subsequent interaction of anionic surfactants and polyelectrolytes with pigment surfaces. Colloids Surf. A: Physicochem. Eng. Aspects. 2002, 203, 21-36.

(81) Li, H.; Tripp, C . P. Spectroscopic identification and dynamics of adsorbed cetyltrimethylammonium structures on $\mathrm{TiO}_{2}$ surfaces. Langmuir 2002, 18, 9441-9446.

(82) Wang, W.; Gu, B.; Liang, L.; Hamilton, W. A. Adsorption and structural arrangement of cetyltrimethylammonium cations at the silica nanoparticle-water interface. J. Phys. Chem. 2004, 108, 17477-17483.

(83) Ström, C.; Hansson, P.; Jönsson, B.; Söderman, O. Size of cationic surfactant micelles at the silica-water interface: A fluorescent probe study. Langmuir 2000, 16, 2469-2474.

(84) Tyrode, E.; Rutland, M. W.; Bain, C. D. Adsorption of CTAB on hydrophilic silica studied by linear and nonlinear optical spectroscopy. J. Am. Chem. Soc. 2008, 130, 1743417445.

(85) Woods, D. A.; Petkov, J.; Bain, C. D. Surfactant adsorption kinetics by Total Internal Reflection Raman spectroscopy. 1. Pure surfactants on silica. J. Phys. Chem. B 2011, 115, 7341-7352.

(86) Torres L. L.; Chauveau M.; Hayes, P. L. Macromolecular structure of dodecyltrimethylammonium chloride at the silica/water interface studied by sum frequency generation spectroscopy. J. Phys. Chem. C 2015, 119, 23917-23927.

(87) Schönhoff, M.; Söderman, O.; Li, Z. H.; Thomas, R. K. Internal dynamics and order parameters in surfactant aggregates: A $2 \mathrm{H}$ NMR study of adsorption layers and bulk phases. Langmuir 2000, 16, 3971-3976.

(88) Schönhoff, M. NMR methods for studies of organic adsorption layers. In: Möbius D, Miller R, editors. Novel methods to study interfacial layers. Amsterdam: Elsevier; 2001. p. 285-336.

(89) Schönhoff, M. NMR studies of sorption and adsorption phenomena in colloidal systems. Curr. Opin. Colloid Interface Sci. 2013, 18, 201-213.

(90) Söderlind, E.; Stilbs, P. A ${ }^{2}$ H NMR study of two cationic surfactants adsorbed on silica particles. Langmuir 1993, 9, 2024-2034.

(91) Totland, C.; Nerdal, W. Thermotropic behavior of a cationic surfactant in the adsorbed and micellar state: An NMR study. Langmuir 2012, 28, 6569-6576.

(92) Wängnerud, P.; Berling, D.; Olofsson, G. Adsorption of alkyltrimethylammonium bromides on silica: Calorimetric study of effect of coions. J. Colloid Interface Sci. 1995, 169, 365-375.

(93) Stodghill, S. P.; Smith, A. E.; O'Haver, J. H. Thermodynamics of micellization and adsorption of three alkyltrimethylammonium bromides using isothermal titration calorimetry. Langmuir 2004, 20, 11387-11392.

(94) Johnson, R. A.; Nagarajan, R. Modeling self-assembly of surfactants at solid liquid interfaces. II. Hydrophilic surfaces. Colloids Surf. A: Physicochem. Eng. Aspects 2000, 167, 21-30. 
(95) Heinz, H.; Castelijns, H. J.; Suter, U. W. Structure and phase transitions of alkyl chains on mica. J. Am. Chem. Soc. 2003, 125, 9500-9510.

(96) Meleshyn, A. Cetylpyridinium chloride at the mica-water interface: Incomplete monolayer and bilayer structures. Langmuir 2009, 25, 881-890.

(97) Schniepp, H. C.; Saville, D. A.; Aksay, I. A. Tip-induced orientational order of surfactant micelles on gold. Langmuir 2008, 24, 626-631.

(98) Walczyk, W.; Schönherr, H. Closer look at the effect of AFM imaging conditions on the apparent dimensions of surface nanobubbles. Langmuir 2013, 29, 620-632.

(99) Berquand, A.; Roduit, C.; Kasas, S.; Holloschi, A.; Ponce, L. Hafner, M. Atomic force microscopy imaging of living cells. Microsc. Today 2010, 18, 8-14.

(100) Heu, C.; Berquand, A.; Elie-Caille, C.; Nicod, L. Glyphosate-induced stiffening of HaCaT keratinocytes, a Peak Force Tapping study on living cells. J. Struct. Biol. 2012, 178, $1-7$.

(101) Pletikapic, G.; Berquand, A.; Radic, T. M.; Svetlicic, V. Quantitative nanomechanical mapping of marine diatom in seawater using PeakForce tapping AFM. J. Phycol. 2012, 48, 174-185.

(102) Walczyk, W.; Schön, P. M.; Schönherr, H. The effect of PeakForce tapping mode AFM imaging on the apparent shape of surface nanobubbles. J. Phys.: Condens. Matter 2013, 25,184005 .

(103) Zhao, B.; Song, Y.; Wang, S.; Dai, B.; Zhang, L.; Dong, Y.; Lü, J.; Hu, J. Mechanical mapping of nanobubbles by PeakForce atomic force microscopy. Soft Matter 2013, 9, 8837-8843.

(104) Nishiyama, T.; Yamada, Y.; Ikuta, T.; Takahashi, K.; Takata, Y. Metastable nanobubbles at the solid-liquid interface due to contact angle hysteresis. Langmuir 2015, 31, 982-986.

(105) Schön, P. M.; Bagdi, K.; Molnar, K.; Markus, P.; Pukanszky, B.; Vancso, G. J. Quantitative mapping of elastic moduli at the nanoscale in phase separated polyurethanes by AFM. Eur. Polym. J. 2011, 47, 692-698.

(106) Adamcik, J.; Berquand, A.; Mezzenga, R. Single-step direct measurement of amyloid fibrils stiffness by peak force quantitative nanomechanical atomic force microscopy. Appl. Phys. Lett. 2011, 98, 193701.

(107) Laughlin, R. G. The aqueous phase behavior of surfactants, Academic Press Ltd, London, 1994.

(108) Di Michele, A.; Brinchi, L.; Di Profio, P.; Germani, R.; Savelli, G.; Onori, G. Effect of head group size, temperature and counterion specificity on cationic micelles. J. Colloid Interface Sci. 2011, 358, 160-166.

(109) Manojlovic, J. Z., The Krafft temperature of surfactant solutions, Therm. Sci. 2012, 16, S631-S640.

(110) Korpelainen, V.; Lassila, A. Calibration of a commercial AFM: traceability for a coordinate system, Meas. Sci. Technol. 2007, 18, 395-403.

(111) Senden, T. J.; Drummond, C. J.; Kékicheff, P. Atomic Force Microscopy: Imaging with electrical double layer interactions. Langmuir 1994, 10, 358-362.

(112) Schönherr, H.; Vancso, G. J. Scanning Force Microscopy of Polymers, Berlin: Springer, 2010.

(113) Claesson, P. M.; Christenson, H. K. Very long range attractive forces between uncharged hydrocarbon and fluorocarbon surfaces in water, J. Phys. Chem. 1988, 92, 1650-1655.

(114) Kékicheff, P.; Cabane, B. Between cylinders and bilayers: structures of intermediate mesophases of the SDS/water system. J. Physique 1987, 48, 1571-1583. 
(115) Kékicheff, P.; Cabane, B. Crystallography of systems with long periods: a neutronscattering study of sodium dodecyl sulfate/water mesophases. Acta Cryst. B 1988, 44, 395-406.

(116) Kékicheff, P. From cylinders to bilayers: A structural study of phase transformations in a lyotropic liquid crystal. Mol. Cryst. Liq. Cryst. 1991, 198, 131-144.

(117) Pagac, E. S.; Prieve, D. C.; Tilton, R. D. Kinetics and mechanism of cationic surfactant adsorption and coadsorption with cationic polyelectrolytes at the silica-water interface. Langmuir 1998, 14, 2333-2342.

(118) Zhang, J.; Yoon, R.-H.; Mao, M.; Ducker, W. A. Effects of degassing and ionic strength on AFM force measurements in octadecyltrimethylammonium chloride solutions. Langmuir 2005, 21, 5831-5841.

(119) Liu, J.-F.; Ducker, W. A. Surface-induced phase behavior of alkyltrimethylammonium bromide surfactants adsorbed to mica, silica, and graphite. J. Phys. Chem. B 1999, 103, $8558-8567$.

(120) Li, B.; Fujii, M.; Fukada, K.; Kato, T.; Seimiya, T. Time dependent anchoring of adsorbed cationic surfactant molecules at mica/solution interface, J. Colloid Interface Sci. 1999, 209, 25-30.

(121) Cantrell, W.; Erwing, G. E. Thin film water on muscovite mica. J. Phys. Chem. B 2001, 105, 5434-5439.

(122) Cheng, L.; Fenter, P.; Nagy, K. L.; Schlegel, M. L.; Sturchio, N. C. Molecular-scale density oscillations in water adjacent to a mica surface. Phys. Rev. Lett. 2001, 87, 156103.

(123) Park, S.-H.; Sposito G. Structure of water adsorbed on a mica surface. Phys. Rev. Lett. 2002, 89, 085501.

(124) Heinz, H.; Vaia, R. A.; Krishnamoorti, R.; Farmer, B. L. Self-assembly of alkylammonium chains on montmorillonite: Effect of chain length, head group structure, and cation exchange capacity. Chem. Mater. 2007, 19, 59-68.

(125) Xu, Y.; Liu, Y.-L.; He, D.-D.; Liu, G.-S. Adsorption of cationic collectors and water on muscovite (001) surface: A molecular dynamics simulation study. Min. Eng. 2013, 53, 101-107.

(126) Xu, Y.; Liu, Y.-L.; He, D.-D.; Liu, G.-S. Molecular dynamics simulation of primary ammonium ions with different alkyl chains on the muscovite (001) surface. Int. J. Miner. Process. 2015, 145, 48-56.

(127) Pashley, R. M.; Quirk, J. P. Ion exchange and interparticle forces between clay surface. Soil Sci. Soc. Am. J. 1989, 53, 1660-1667.

(128) Trompette, J. L; Zajac, J.; Keh, E.; Partyka, S. Scanning of the cationic surfactant adsorption on a hydrophilic silica surface at low surface coverages. Langmuir 1994, 10, 812-818.

(129) Doudevski, I.; Hayes, W. A.; Woodward, J. T.; Schwartz, D. K. Atomic force microscope imaging of molecular aggregation during self-assembled monolayer growth. Colloids Surf. A 2000, 174, 233-243.

(130) Kékicheff, P.; Grabielle-Madelmont, C.; Ollivon, M. Phase diagram of sodium dodecyl sulfate - water system: 1. A calorimetric study. J. Colloid Interface Sci. 1989, 131, $112-$ 132.

(131) Kékicheff, P. Phase diagram of sodium dodecyl sulfate - water system: 2. Complementary isoplethal and isothermal phase studies. J. Colloid Interface Sci. 1989, $131,133-152$.

(132) Rabinovich, Y. I.; Vakarelski, I. U.; Brown, S. C.; Singh, P. K.; Moudgil, B. M. Mechanical and thermodynamic properties of surfactant aggregates at the solid-liquid interface. J. Colloid Interface Sci. 2004, 270, 29-36. 
(133) Chiu, P. Y.; Shah, K.; Sinnott, S. B. Nanoindentation of surfactant aggregates. J. Colloid Interface Sci. 2010, 349, 196-204.

(134) Velegol, S. B.; Pardi, S.; Li, X.; Velegol, D.; Logan, B. E. AFM imaging artifacts due to bacterial cell height and AFM tip geometry. Langmuir 2003, 19, 851-857.

(135) Golek, F.; Mazur, P.; Ryszka, Z.; Zuber, S. AFM image artifacts. Appl. Surf. Sci. 2014, 304, 11-19.

(136) Shen, J.; Zhang, D.; Zhang, F.-H.; GAn, Y. AFM characterization of patterned sapphire substrate with dense cone arrays: Image artifacts and tip-cone convolution effects. Appl. Surf. Sci. 2018, 433, 358-366.

(137) Canet-Ferrer, J.; Coronado, E.; Forment-Aliaga, A.; Pinilla-Cienfuegos, E. Correction of the tip convolution effects in the imaging of nanostructures studied through scanning force microscopy. Nanotechnology 2014, 25, 395703.

(138) "Guide to the Expression of Uncertainty in Measurement", Joint Committee for Guides in Metrology, JCGM 104 (2009).

(139) Christenson, H. K.; Claesson, P. M. Direct measurements of the force between hydrophobic surfaces in water. Adv. Colloid Interface Sci. 2001, 91 (3), 391-436. 
Abstract Graphic (TOC)
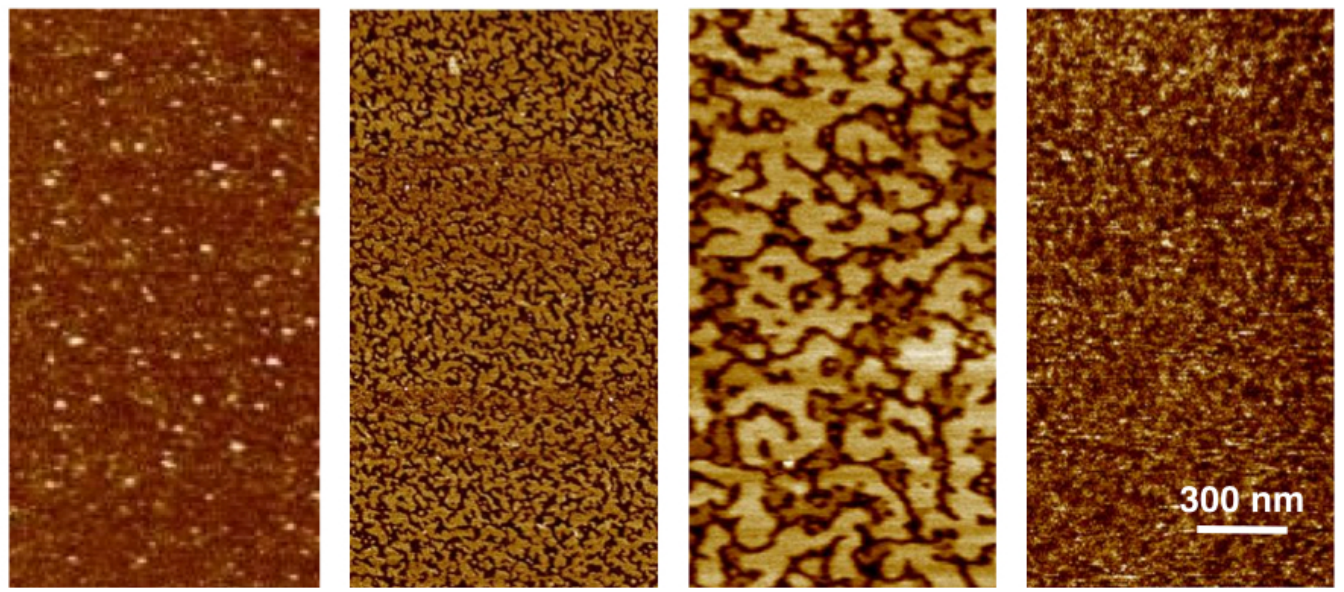

$\mathrm{C}_{16}$ TAB concentration 